\title{
STAR FORMATION RATE DISTRIBUTIONS: INADEQUACY OF THE SCHECHTER FUNCTION
}

\author{
SAMIR SALim ${ }^{1}$ AND JANICE C. LeE 2,3 \\ ${ }^{1}$ Department of Astronomy, Indiana University, Bloomington, IN 47404, USA; salims@ indiana.edu \\ 2 Space Telescope Science Institute, Baltimore, MD 21218, USA \\ Received 2012 July 13; accepted 2012 September 7; published 2012 October 9
}

\begin{abstract}
In this paper, we posit that galaxy luminosity functions (LFs) come in two fundamentally different types depending on whether the luminosity traces galaxy stellar mass or its current star formation rate (SFR). Mass function types reflect the older stars and therefore the stellar mass distribution, while SFR function types arise from the young stars and hence the distribution of SFRs. Optical and near-infrared LFs are of the mass function type and are well fit by a Schechter function (power law with an exponential cutoff at the bright end). In contrast, LFs of the SFR function type are of a different form, one that cannot be adequately described by a Schechter function. We demonstrate this difference by generating SFR distributions for mock samples of galaxies drawn from a Schechter stellar mass distribution along with established empirical relations between the SFR and stellar mass. Compared with the Schechter function, SFR distributions have a shallower decline at the bright end, which can be traced to the large intrinsic scatter of SFRs at any given stellar mass. A superior description of SFR distributions is given by the "Saunders" function, which combines a power law with a Gaussian at the high end. We show that the Schechter-like appearance of UV and $\mathrm{H} \alpha$ LFs, although they are LFs of SFR function type, results when luminosities are not corrected for dust, or when average statistical corrections are used because individual attenuation measurements are not available. We thus infer that the non-Schechter form of the far-IR LFs is a true reflection of the underlying SFR distribution, rather than the purported artifact of active galactic nucleus contamination.
\end{abstract}

Key words: galaxies: evolution - galaxies: fundamental parameters - galaxies: luminosity function, mass function - methods: analytical - methods: numerical

Online-only material: color figures

\section{INTRODUCTION}

Schechter (1976) realized that the distribution of optical luminosities of cluster galaxies empirically follows the same functional form that has been introduced on theoretical grounds by Press \& Schechter (1974) to describe the halo mass function (MF). This functional form is now known as the Schechter function. It combines a power law at the faint end with an exponential cutoff at the bright end and is uniquely determined with three parameters. The function has been shown to describe luminosities of galaxies in field environments too (Felten 1977). Parameterization of the optical luminosity function (LF) using a Schechter function has simplified comparisons of different samples of galaxies, including samples at different redshifts, and the determination of the cosmic luminosity density (e.g., Binggeli et al. 1988).

With the advent of multiwavelength galaxy surveys, LFs began to be constructed in the far-IR (e.g., Lawrence et al. 1986), near-IR (e.g., Mobasher et al. 1993), and UV (e.g., Sullivan et al. 2000), as well as for optical emission line luminosities (e.g., Gallego et al. 1995). It was generally expected that these LFs will also follow the Schechter function, and most of these studies, oftentimes using LFs with very limited dynamic range, confirmed such expectations. One striking exception was the LF in the far-IR (Lawrence et al. 1986; Saunders et al. 1990), which was possible to construct over a very wide dynamic range $(\sim 5$ dex in space density) and showed a significantly shallower decline at the bright end than the exponential decline of the Schechter function. While this difference between far-IR and

\footnotetext{
3 Visiting Astronomer, Spitzer Science Center, Caltech, Pasadena, CA 91125,
} USA. other LFs has been acknowledged (e.g., Buat \& Burgarella 1998; Takeuchi et al. 2005), the expectations set by the perceived ubiquitousness of the Schechter distribution led some to consider the far-IR LF as anomalous and perhaps deviating from the Schechter form because of an active galactic nucleus (AGN) contamination (e.g., Bothwell et al. 2011).

In this paper, we show that there is a different explanation for such deviations from the Schechter form. We propose that there are two fundamentally different galaxy distribution functions: (1) of the stellar mass (the $\mathrm{MF}^{4}$ ) and of the (2) star formation rate (the SFR function). LFs in different parts of the spectrum will be related more to one type or the other. Optical (especially in bands past the $4000 \AA$ break) and near-IR luminosities arise from lower-mass stars that contain most of the stellar mass; therefore, these LFs belong to the MF type. On the other hand, the emission in the UV, the nebular line emission (e.g., $\mathrm{H} \alpha$, [O II] $)$, and the thermal IR are more closely related to young stellar populations. Therefore, such LFs, if properly dust corrected, should belong to the SFR function type.

What are the true underlying functional forms of the stellar MF and the SFR function? Are they different? In the last decade advances in stellar population modeling and the availability of large surveys made the determination of the galaxy stellar masses possible for a large number of galaxies. Being more fundamental than the optical LF, the MF received prompt attention. It too was found to follow Schechter's functional form (Cole et al. 2001; Bell et al. 2003). Indeed, it can be said that the LFs in the optical and the near-IR reflect the underlying Schechter-like distribution of stellar masses.

\footnotetext{
4 Since we will only be discussing the stellar mass, we will often be omitting the adjective "stellar."
} 
Accurate SFR functions are more difficult to construct than the MFs due to the caveats and larger uncertainties involved in deriving SFRs (e.g., Kennicutt 1998). As pointed out, some LFs of SFR type were found to be consistent with a Schechter form (UV and $\mathrm{H} \alpha$ ), while others, most notably the far-IR, were not. The current literature has not fully explained this difference. The role of dust has been implied in, e.g., Buat \& Burgarella (1998), Martin et al. (2005), Takeuchi et al. (2005), and Reddy et al. (2010). A related question, why LFs from young stars (UV and $\mathrm{H} \alpha$ ) appear to have the same Schechter-like distribution as LFs dominated by old stars (optical and near-IR), received even less attention.

This study sets out to determine the intrinsic form of the SFR function (in turn testing the adequacy of the Schechter function) and to explain why different tracers produce different results. Knowing the appropriate parametric form of the SFR function will help interpret the observations at the range of redshifts and will facilitate comparison with galaxy formation simulations.

To carry out the search for the functional form of the SFR function (SFRF), we adopt a simple framework in which we produce volume-complete mock samples of galaxies (Section 2.1) that are described by two quantities: stellar mass and SFR, where the stellar mass is drawn from a Schechter function, while the SFR is obtained by empirically motivated relations between mass and SFR. We apply a series of three such SFR-mass relations of increasing complexity, the final of which being a relatively realistic representation of the observed SFR-mass plane. We then study the SFR distributions produced by each relation and discuss functional forms that can be used to describe them (Sections 2.2-2.4). Readers not interested in the details of these exercises should skip to the summary in Section 2.5. We conclude that Schechter formulation is not adequate for describing the SFR function. Instead, functions that replace the exponential function at the high end with a Gaussian represent a far better description. Next, in Section 3 we discuss the implications of the use of non-Schechter functions for the derivation of the SFR density. In Section 4, we apply the proposed functional forms to the observed local SFRF and find excellent agreement. In Section 5, we discuss the observed properties of LFs of SFR type (UV, $\mathrm{H} \alpha$, and IR) and provide explanation as to why UV and $\mathrm{H} \alpha$ LFs appear to be well described using the standard Schechter function despite being forms of an SFR function. We show that the Schechter-like behavior is a coincidence stemming from the non-application of dust corrections (or from application of only average statistical corrections for dust) instead of dust corrections based on more robust measurements of the attenuation on an individual galaxy basis. On the other hand, we show that the non-Schechter form of far-IR LFs more closely reflects the true SFR function and is not the result of a purported AGN contamination.

Cosmological parameters $\Omega_{m}=0.3, \Omega_{\Lambda}=0.7$, and $H_{0}=$ $70 \mathrm{~km} \mathrm{~s}^{-1} \mathrm{Mpc}^{-1}$ are assumed throughout. We express all stellar masses and SFRs assuming the Chabrier initial mass function (IMF).

\section{SFR FUNCTION}

The goal of this paper is to evaluate the adequacy of the Schechter function for describing the SFRF and to propose eventual alternatives to this function. In order to perform such an evaluation, the "true" expected SFRF must be known. We derive the expected SFRF from the combination of two relations that are well determined locally: the stellar MF and the stellar mass-SFR relation.
To produce SFR functions, we construct mock samples in the following way: we draw a large sample from an MF that follows Schechter parameterization. Then, to each mock galaxy we associate an SFR based on an SFR-mass relation. We explore three different types of stellar SFR-mass relations: (1) simple power-law relation between SFR and mass with no scatter, (2) power-law relation with scatter, and (3) bimodal powerlaw relation with scatters in both modes. As discussed below, each succeeding relation is meant to be more realistic than the previous. The last should come very close to describing true SFRs. For SFRFs resulting from these relations we test the adequacy of the Schechter function and search for other functional forms that potentially describe them better.

\subsection{Construction of Mock Samples}

To define the underlying Schechter MF from which the mock samples of galaxies are drawn, we adopt parameters from Panter et al. (2004), who present an MF based on the Sloan Digital Sky Survey (SDSS) spectroscopic sample. For our choice of Hubble constant and IMF these Schechter parameters have the following values: MF normalization $\phi^{\star}=2.7 \times 10^{-3} \mathrm{Mpc}^{-3} \mathrm{dex}^{-1}$, characteristic mass (converted from Salpeter IMF by dividing the mass by 1.228 , based on Bruzual \& Charlot 2003 models), $\log M_{*}^{\star}=11.10$ (in solar mass units throughout), and the faintend slope exponent of $\alpha=-1.16$. This MF was constructed using data with $\log M_{*} \gtrsim 7.7$, but we verify that its Schechter function fit is in excellent agreement with the latest $6<$ $\log M_{*}<8 \mathrm{MF}$ measurements from Baldry et al. (2011), which means that it can be safely extrapolated to lower masses.

From this MF we draw two volume-complete mock samples: (1) a mass-limited sample with $M_{*}>10^{8} M_{\odot}$ and (2) an SFRlimited sample with SFR $>0.01 M_{\odot} \mathrm{yr}^{-1}$. SFRs are assigned to each galaxy according to one of the three SFR-mass relations as previously described. Distinguishing between the two samples with different types of limits is important because each affects the shape of the SFR function differently.

The exact choice of limits has no consequence on the inferences drawn from the analysis, but we wish that they reflect some realistic scenarios. For the mass-limited sample we take the limit to be $\log M_{*}=8$, which is approximately the lowest mass for which statistically large samples can be extracted from SDSS (e.g., Baldry et al. 2008). To this sample we do not impose any limits in terms of SFR. For the SFR-limited sample we take the limit of $0.01 M_{\odot} \mathrm{yr}^{-1}$, but no mass limit. Throughout this work SFRs represent true, dust-corrected SFRs. This SFR limit matches the completeness limits of surveys that target very nearby galaxies (such as the Local Volume Legacy survey; Kennicutt et al. 2008; Dale et al. 2009; Lee et al. 2011). Most of the galaxies that produce stars at the rate around the limit are dwarfs with $6<\log M_{*}<8$ (Johnson 2011). This SFR-limited sample will probe a fraction of actively starforming dwarfs at that mass and principally all star-forming galaxies above $\log M_{*}=8$ (Section 2.3). In the local universe, this SFR limit probes $99 \%$ of the total SFR density (Section 3).

The volume for the mock samples is $10^{8} \mathrm{Mpc}^{3}$ and was chosen to be large enough so that the features of the SFR function are not significantly affected by the Poisson noise over a large dynamic range in space density $(\sim 5 \mathrm{dex})$. The mass-limited sample contains $3.4 \times 10^{6}$ galaxies, and the SFR-limited sample up to $7.0 \times 10^{6}$, depending on the SFR-mass relation used. Unlike real surveys, our samples are volume-complete by construction, so no completeness corrections are required (for reviews of the 

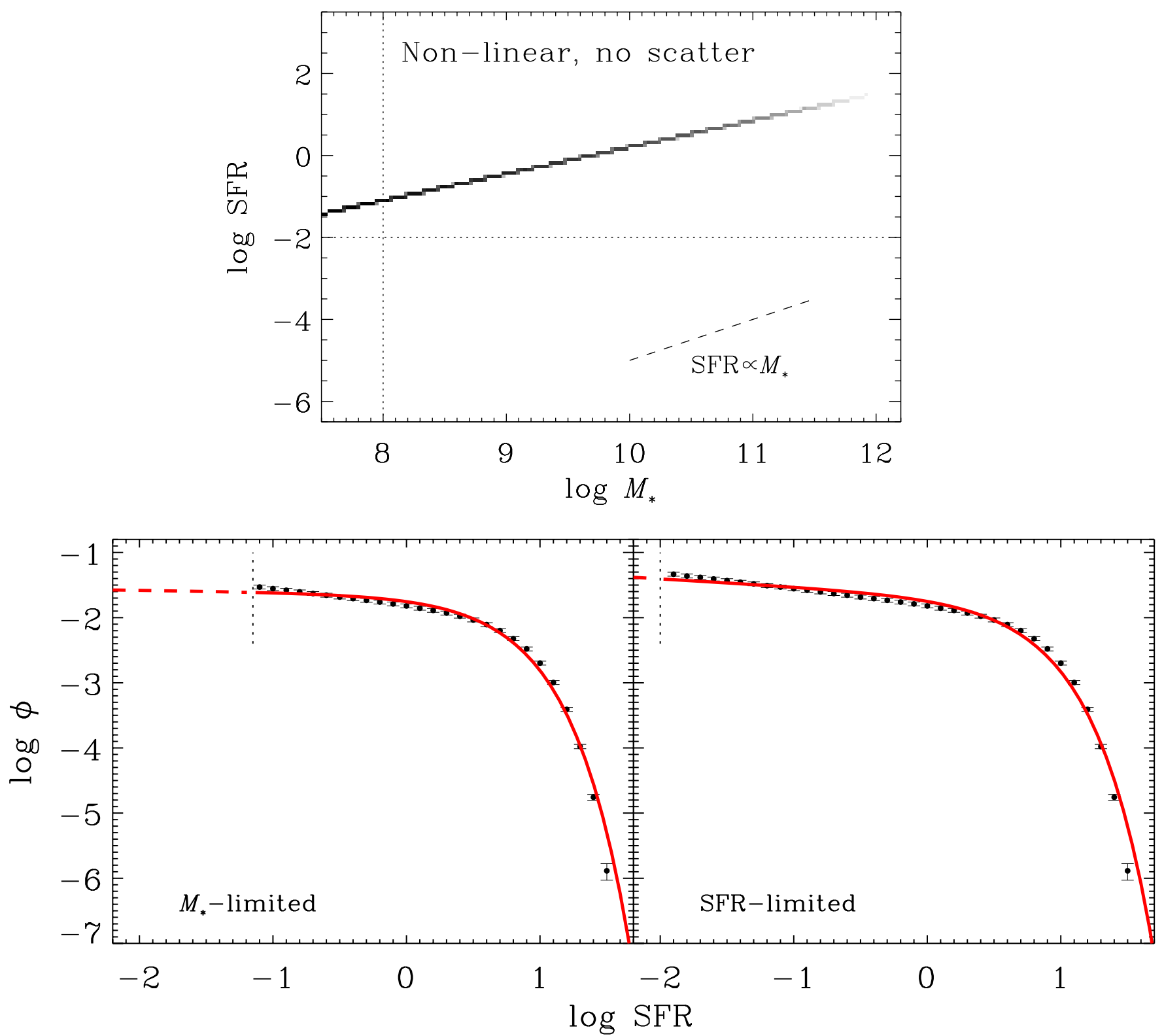

Figure 1. Relation (1) and the resulting SFR functions. SFR scales as a sub-linear power of $M_{*}$, with no scatter, the simplest of the three relations that we explore. Upper panel shows the dependence of SFR on stellar mass. The relation appears jagged because it is represented as the bivariate density image, which is pixelated. The underlying stellar mass function is assumed to have the Schechter form. Dotted lines show limits for mass- and SFR-limited samples. Lower panels show the resulting SFR functions for the mass-limited (left, $\log M_{*}>8$ ) and the SFR-limited samples (right, $\log$ SFR $>-2$ ). Vertical dashed lines indicate the lower cutoff used in the fitting. Red curves represent the best-fitting Schechter functions. Neither Schechter fit describes the SFRF accurately.

(A color version of this figure is available in the online journal.)

construction of the observed LFs, see Johnston 2011; Takeuchi et al. 2000; Willmer 1997).

\subsection{Relation 1: SFR Scales as a Power of $M_{*}$ with No Scatter}

Recent studies at low (e.g., Boselli et al. 2001; Brinchmann et al. 2004; Salim et al. 2007) and intermediate redshifts (e.g., Noeske et al. 2007; Elbaz et al. 2007) have found that for actively star-forming galaxies there is a relatively tight and straight sequence in $\log M_{*}$ versus $\log$ SFR space, which has been dubbed the star-forming sequence (Salim et al. 2007) or the galaxy main sequence (Noeske et al. 2007). Straight sequence in log space is equivalent to a power-law relation between SFR and mass ( $\mathrm{SFR} \propto M_{*}^{\beta}$ ). The reasons behind the existence of the relation are currently the focus of many theoretical studies (e.g., Dutton et al. 2010; Davé et al. 2011).

Here, we adopt the empirical relation derived in the local universe $(z \sim 0.1)$ from Salim et al. (2007) (Equation (11)):

$$
\log \mathrm{SFR}=0.65 \log M_{*}-6.33,
$$

where masses and dust-corrected SFRs were obtained through the use of UV/optical spectral energy distribution (SED) fitting of Galaxy Evolution Explorer (GALEX) and SDSS fluxes of galaxies falling in the star-forming part of the BPT diagram (Baldwin et al. 1981). The relation is shown in Figure 1 (upper panel) and is sub-linear $(\beta=0.65)$. Other studies have found different values of $\beta$, but they are usually sub-linear (Dutton et al. 2010; Huang et al. 2012). 
Equation (1) is applied to masses drawn from the Schechter MF to obtain their corresponding SFRs. SFRs are then binned in 0.1 dex intervals to obtain SFR functions, shown in Figure 1 (lower panels). Error bars represent Gaussian approximation of the Poisson error and are typically extremely small for most of the bins, meaning that the features of SFRFs are accurately determined. To ensure more even weighting when performing the fitting, we add in each bin 0.03 dex (7\%) of systematic error, similar to errors in well-determined empirical LFs (Blanton et al. 2001).

What functional form best describes the SFR functions in Figure 1 (lower panels)? Their appearance suggests that they would be well fit with a standard Schechter function: ${ }^{5}$

$$
\Phi_{\mathrm{S}}(X) d X=\frac{\phi^{\star}}{X^{\star}}\left(\frac{X}{X^{\star}}\right)^{\alpha} e^{-X / X^{\star}} d X
$$

where $X=\mathrm{SFR}, X^{\star}$ is the characteristic SFR, $\phi^{\star}$ is the normalization (expressed in units of $\mathrm{Mpc}^{-3} \mathrm{dex}^{-1}$ or $\mathrm{Mpc}^{-3} \mathrm{mag}^{-1}$ throughout), and $\alpha$ is the "faint"-end power-law exponent. We show the best-fitting Schechter function, obtained by minimizing $\chi^{2}$, as solid lines in Figure 1 (lower panels). ${ }^{6}$ The Schechter fits do not follow the SFRFs exactly. The low-SFR ("faint" end) slope of both fits tends to be shallower than the SFRF points. Similarly, the knee of the fits appears to lie at lower SFRs than what is expected visually. Finally, at the high end the fits are slightly shallower than the SFR function. This mismatch is corroborated with the large $\chi^{2}$ per degree of freedom (reduced $\chi^{2}$ ) values of $\chi_{r}^{2}=6.2$ and 7.0 for the mass- and SFR-limited cases, respectively. Why is the Schechter function not a perfect fit as may perhaps be expected?

The answer is that after the power-law transformation, the exponential part of the Schechter function becomes modified into a Sérsic function. Unlike the exponential function, which has a fixed high-end slope, the Sérsic function will have different slopes based on the extra parameter that is featured in it. The reason why the SFR function constructed in this way appears to be a Schechter function is because on a logarithmic plot the shapes of exponential and Sérsic functions are identical modulo the scale factor, i.e., we can always pick an $x$ scale such that the two shapes are exactly the same.

To properly fit the SFR distribution constructed using Equation (1), the Schechter function needs to be modified by introducing an additional parameter: the power-law exponent $\beta$ between the mass and SFR. We call this the extended Schechter function: ${ }^{7}$

$$
\Phi_{\mathrm{ES}}(X) d X=\frac{1}{\beta} \frac{\phi^{\star}}{X^{\star}}\left(\frac{X}{X^{\star}}\right)^{\alpha^{\prime}} \exp \left[-\left(X / X^{\star}\right)^{1 / \beta}\right] d X .
$$

The exponential part of the standard Schechter function became the Sérsic function, with $\beta$ being equivalent to the Sérsic index. The extended Schechter function is the regular Schechter function when $\beta=1$. We confirm that the extended Schechter function fits the values of the SFR function perfectly and retrieves parameters of the generating MF and SFR-mass

\footnotetext{
5 The logarithmic form of the standard Schechter function is expressed as $\Phi_{\mathrm{S}}(\log X) d(\log X)=$

$\ln (10) \phi^{\star} 10^{(\alpha+1)\left(\log X-\log X^{\star}\right)} \exp \left[-10^{\left(\log X-\log X^{\star}\right)}\right] d(\log X)$.

6 Fitting is done in $\log \phi$.

7 The logarithmic expression for the extended Schechter function is $\Phi_{\mathrm{ES}}(\log X) d(\log X)=$

$\ln (10) \frac{\phi}{\beta}^{\star} 10^{\left(\alpha^{\prime}+1\right)\left(\log X-\log X^{\star}\right)} \exp \left[-10^{\left(\log X-\log X^{\star}\right) / \beta}\right] d(\log X)$.
}

relation. ${ }^{8}$ We do not show these fits in Figure 1 (lower panels) since they would simply pass through all the points with zero deviation.

The need for extending the Schechter function in order to model certain distribution functions has recently been recognized in Bernardi et al. (2010), following Sheth et al. (2003), and in Hopkins et al. (2010). Bernardi et al. (2010) note that galaxy sizes and velocity dispersions scale as power laws with respect to the optical luminosity $(\phi(L))$ and therefore remark that "if $\phi(L)$ is well fit by a Schechter function, it makes little physical or statistical sense to fit the other observables with a Schechter function as well." ${ }^{9}$ Hopkins et al. (2010) is the only work to our knowledge that has attempted to apply the extended Schechter formulation to an SFR-like distribution (a simulated IR LF).

The relation between mass and SFR assumed in this section is very simplistic, so even the extended Schechter function will not accurately reproduce all the features of real SFR distributions. Nevertheless, it already demonstrates that SFR functions cannot be adequately described by Schechter functions.

\subsection{Relation 2: SFR Scales as a Power of $M_{*}$ with Scatter}

While the SFR versus mass relation is relatively tight, any scatter around this relation that is not correlated with the mass would affect the shape of the resulting SFR function. We model this scatter with a Gaussian (in log SFR) of $\sigma=0.4$ dex, again based on the results from Salim et al. (2007). ${ }^{10}$ The scatter along the star formation (SF) sequence increases with mass from 0.3 to $0.4 \mathrm{dex}$, but the constant value is a reasonable approximation for this exercise. This scatter is predominantly intrinsic, since the SFR errors in Salim et al. (2007) for galaxies on the SF sequence are $\approx 0.2$ dex. The SFR-mass relation with scatter is shown in Figure 2 (upper panel).

Figure 2 (lower panels) shows the SFR functions for the massand SFR-limited samples. There are several notable differences of these SFRFs with respect to the case with no scatter. At the high end the tail is now much less steep, extending to higher SFRs (note that the horizontal scale in Figure 2 (lower panels) is much wider than in Figure 1 (lower panels)), so the knee appears "softer." The mass- and SFR-limited SFRFs start to differ significantly at $\log$ SFR $\lesssim-0.5$. The SFR function of the mass-limited sample (Figure 2 (lower left panel)) now features a turnover and drops off at the low end. This feature is due to the tail of galaxies close to the low-mass limit that scatter below the mean SFR $-M_{*}$ relation. The falloff is obviously a mathematical consequence of the presence of the mass limit, but it is easy to confuse it with volume incompleteness, which is why we show its effects separately. For the sample with no mass cut (Figure 2 (lower right panel)) there is no such turnover and the SFRF continues to rise. Knowing how the sample is selected is therefore very important in interpreting the "faint" end of any observed SFR function. The falloff at the low SFRs can also be seen in the cosmological simulations of the SFR function of Davé et al. (2011) and the semi-analytic modeling

\footnotetext{
8 The low-end slope in the extended Schechter function is related to the low-end slope of the generating MF, which features in Equation (2) as $\alpha=\beta\left(\alpha^{\prime}+1\right)-1$.

9 The Bernardi et al. (2010) formulation of the extended Schechter function (their Equation (9)) appears to have an error (what is listed as $1 / X$ should be $\left.1 / X^{\star}\right)$. Furthermore, $\beta$ in Bernardi et al. (2010) and Hopkins et al. (2010) is the slope of mass versus $X$ and therefore the inverse of our $\beta$.

10 Throughout this paper, we will refer to Gaussians, keeping in mind that in the linear SFR these functions are actually lognormal.
} 

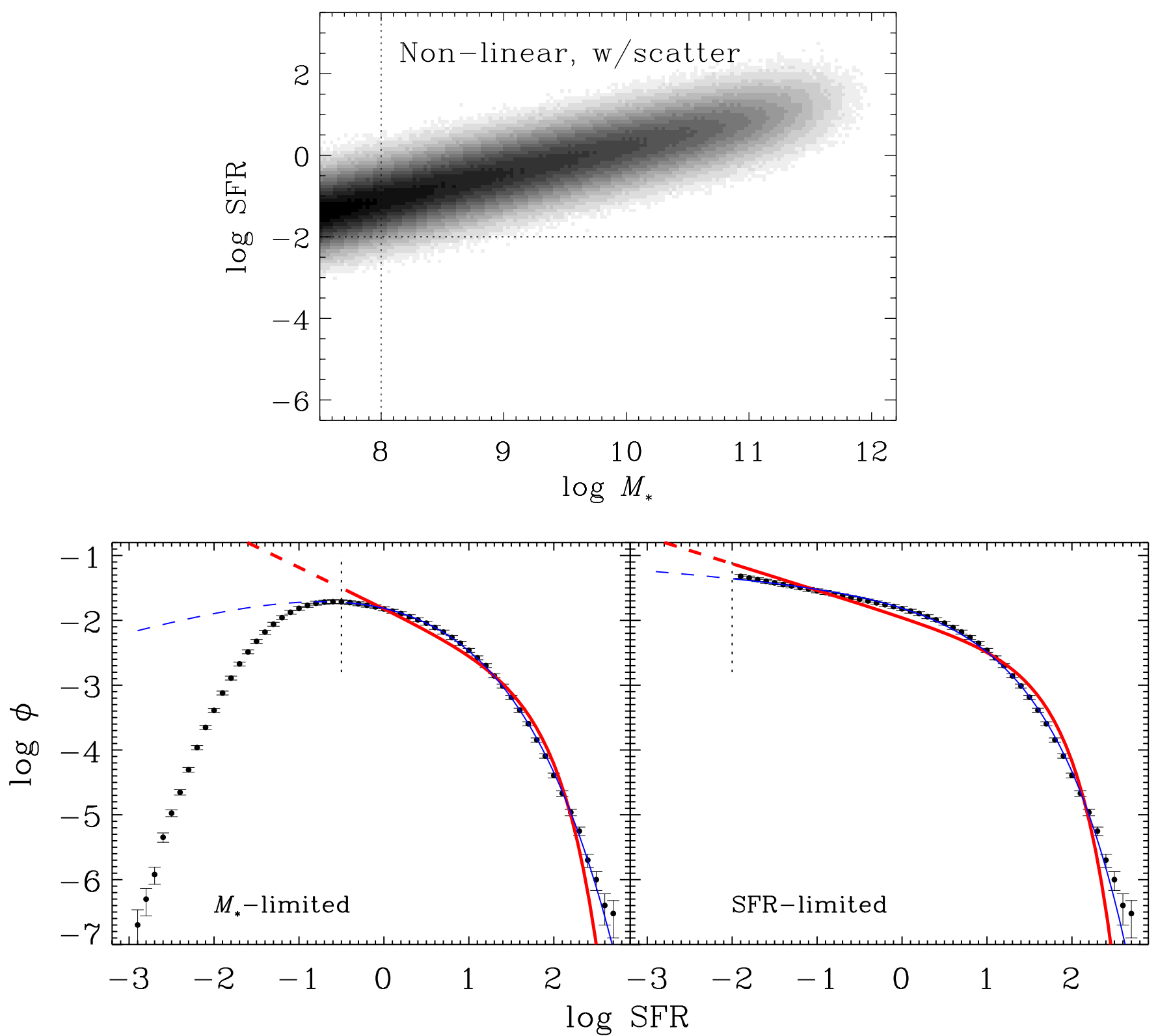

Figure 2. Relation 2 and the resulting SFR functions. SFR scales as the power of $M_{*}$, with a Gaussian scatter of 0.4 dex in log SFR (lognormal in linear SFR). The upper panel shows the dependence of SFR on the stellar mass. Dotted lines show the limits of mass- and SFR-limited samples. Lower panels show the resulting SFR functions for the mass-limited (left, $\log M_{*}>8$ ) and the SFR-limited samples (right, $\log$ SFR $>-2$ ). Vertical dashed lines indicate the lower limits used in fitting. Thick curves (red) represent the best-fitting Schechter functions, which describe the distributions very poorly. Thin curves (blue) are the best-fitting extended Schechter functions, which yield very good fits in the fitted regions.

(A color version of this figure is available in the online journal.)

of Fontanot et al. (2012), with both groups using mass limits in their simulations.

In evaluating the functional forms that could be used to describe these SFR functions, we again start with the regular Schechter function (Equation (2)). For the mass-limited sample (Figure 2 (lower left panel)) we limit the fitting to the part higher than the turnover $(\log S F R \geqslant-0.5$, dotted vertical line), since obviously the Schechter function (or the extended Schechter function) will not be able to reproduce the drop at low values. The red line shows the best fit. One can see that the shape of the Schechter function is quite inadequate, and the resulting parameters are consequently of little value. The regular Schechter function does not perform much better for the case of the SFR-limited sample either (red line in Figure 2 (lower right panel), with both the low-end slope and the high-end drop too steep and the knee too high. Formal reduced $\chi_{r}^{2}$ values are in both cases extremely large (17 and 23, respectively). Note, however, that in some real data sets where the data points have significantly larger error bars and the dynamic range is small, a Schechter function fit could be formally acceptable, leading one to believe that the distribution is intrinsically of the Schechter form.

On the other hand, the extended Schechter fit brings significant improvements in describing both samples (blue lines in Figure 2 (lower panels)), with $\chi_{r}^{2}=0.3$ and 1.2 for the massand SFR-limited distributions, respectively. Note, however, that even the extended Schechter function cannot reproduce these SFRFs perfectly. The consequence is that the best-fit parameters 
cannot be directly mapped back to parameters of the underlying mass distribution and the relationship between the mass and the SFR. In the SFR-mass relation without scatter, $\beta$ represented the slope of the SFR-mass relation. Now, the best fits have $\beta$ values of 2.08 and 3.03 for the mass- and SFR-limited distribution, respectively, in contrast with SFR-mass slope of 0.65 .

The only way to reproduce these SFR functions exactly would be to again reverse the process by which the SFRs were constructed. This can be achieved with the extended Schechter function convolved with a Gaussian. Such a "function" would feature five or six parameters: four of the extended Schechter functions, the scatter $\sigma$, and also the mass limit for mass-limited samples. We confirm that this function fits SFRFs in Figure 2 (lower panels) perfectly (fits not shown), with the resulting parameters again having an interpretable meaning. However, for any SFRF based on real data the cost of two to three additional parameters with respect to the number needed to describe the Schechter function will be too large-the resulting fits would suffer from a high degree of degeneracy and we will consequently not consider this construct in further analysis.

Bernardi et al. (2010) provided an approximate analytical expression for the effect of the measurement error on the extended Schechter function in the limit of small $\sigma$ (their Equations (10) and (11)). However, that form is not appropriate for the level of scatter encountered here, which is dominated by large intrinsic scatter. This is exemplified by the fact that the distribution corrected in that way does not preserve the total number density of galaxies since the correction factor is always greater than one.

\subsection{Relation 3: SFR Is Bimodal, Each Mode Scales as a Power of $M_{*}$ with Scatter}

After including the scatter in the SFR-mass relationship, we now add one final element to bring mock SFRs close to the realistic ones: galaxy bimodality. Galaxy bimodality is most often used to describe the character of optical color distribution. The blue mode of the color distribution corresponds to the starforming sequence, which is what was modeled in the previous sections. The optical red mode corresponds to galaxies that do not belong to the star-forming sequence and thus have little or no SF. We refer to them as passive galaxies. They include optically red galaxies with SFRs measurably different from zero (e.g., the green valley galaxies detected using UV-optical colors; Martin et al. 2007; Salim et al. 2007) and the galaxies with upper limits on SFR consistent with no SF.

If the passive galaxies were taken to have exactly zero SFR, then the modeling of the SFR distribution reverts to the unimodal case (Section 2.3), with the only difference being that the underlying MF would be for blue (star-forming) galaxies alone. However, it is more realistic to characterize passive galaxies with a range of non-zero SFRs, especially since passive galaxies on the massive end can reach relatively high SFRs $\left(\lesssim 1 M_{\odot} \mathrm{yr}^{-1}\right.$; Cortese 2012).

To specify bimodal SFR-mass relations for use in our modeling, we again draw on the data derived in Salim et al. (2007). Expressions for separate SF and passive sequences were not given in that paper, so we determine them now by fitting in each mass bin two Gaussians in log SFR. We find that the modeling of the SFR distribution at a given mass with two Gaussians describes these distributions remarkably well. Unlike in optical color where the colors of passive galaxies quickly saturate (the red sequence), the passive sequence is quite broad in $\log$ SFRs, resulting in peaks that are not well separated, with no pronounced dip between them. The data probe the passive sequence very well by reaching down to $\log \left(\mathrm{SFR} / M_{*}\right)=-14$. The peaks of Gaussians yield the following SFR-mass relations that are very well described with power laws. For the SF sequence

$$
\log \mathrm{SFR}=0.54 \log M_{*}-5.42,
$$

which is slightly shallower than the SF sequence defined by SF galaxies selected using the BPT diagram (Equation (1)), and for the passive sequence

$$
\log \mathrm{SFR}=0.38 \log M_{*}-5.20,
$$

the scatter of which varies from $1.5 \mathrm{dex}$ at $\log M_{*} \sim 9$ to $0.7 \mathrm{dex}$ at $\log M_{*} \sim 11.8$ and is again a combination of measurement errors and, to a larger extent, the intrinsic scatter. For simplicity, in our modeling we take the scatter of the passive sequence to be fixed at 1.1 dex, while for the SF sequence we use a scatter of 0.4 dex as previously.

At each mass, we determine the passive fraction from the ratio of the area below the Gaussian of the passive sequence and the total areas of both Gaussians, which can be obtained from

$$
f_{\text {pass }}=\frac{N_{\text {pass }} \sigma_{\text {pass }}}{N_{\text {pass }} \sigma_{\text {pass }}+N_{\mathrm{SF}} \sigma_{\mathrm{SF}}},
$$

where $N$ is the height of the peaks. It increases from around $30 \%$ at $\log M_{*}=8$ to $80 \%$ at $\log M_{*}=11.5$. The passive fraction is well described as a quadratic function of mass:

$$
f_{\text {pass }}=0.0534 \log ^{2} M_{*}-0.905 \log M_{*}+4.144 .
$$

In Figure 3 (upper panel), we show the SFR versus mass values of the simulated bimodal distribution. It was constructed so that for each galaxy we first determine if it is passive or active using a random number and Equation (7). Then, we draw SFRs from the appropriate sequence and add the corresponding scatter. Since the passive sequence has a fairly large scatter, we do not allow a passive galaxy to have an SFR greater than the SF sequence (Equation (1)) increased by $1 \sigma$ (i.e., 0.4 dex). In such cases we draw a new value for the SFR. For the mass-limited sample a full span in mock SFRs is now $\approx 9$ orders of magnitude.

The resulting SFR functions are shown in Figure 3 (lower panels) for the mass- and SFR-limited samples, respectively. The two distributions start to depart below $\log$ SFR $\approx 0$. The main difference of the bimodal mass-limited SFRF compared to its unimodal counterpart is an even more gradual drop to lower values due to the relatively low SFRs of the low-mass passive galaxies and the presence of the inflection point (the "dip") at $\log$ SFR $\approx-1$, reflecting the bimodal nature of the SFR distribution at each mass. On the other hand, the SFR-limited distribution remains monotonic because at each SFR it is dominated by galaxies in the star-forming sequence.

We again seek an appropriate functional fit for these SFR functions, beginning with the SFR-limited distribution. Since the SFR-limited distribution is very similar in the bimodal and unimodal case, the regular Schechter function can be ruled out as a satisfactory form based on previous considerations. As in the case of the unimodal distribution, the extended Schechter function represents an excellent fit $\left(\chi_{r}^{2}=0.3\right)$. We show it in Figure 3 (lower right panel) with a blue line.

While the extended Schechter function represents an excellent functional form for fitting the SFR-limited SFRF, recall that its introduction was motivated by "inverting" the power-law 

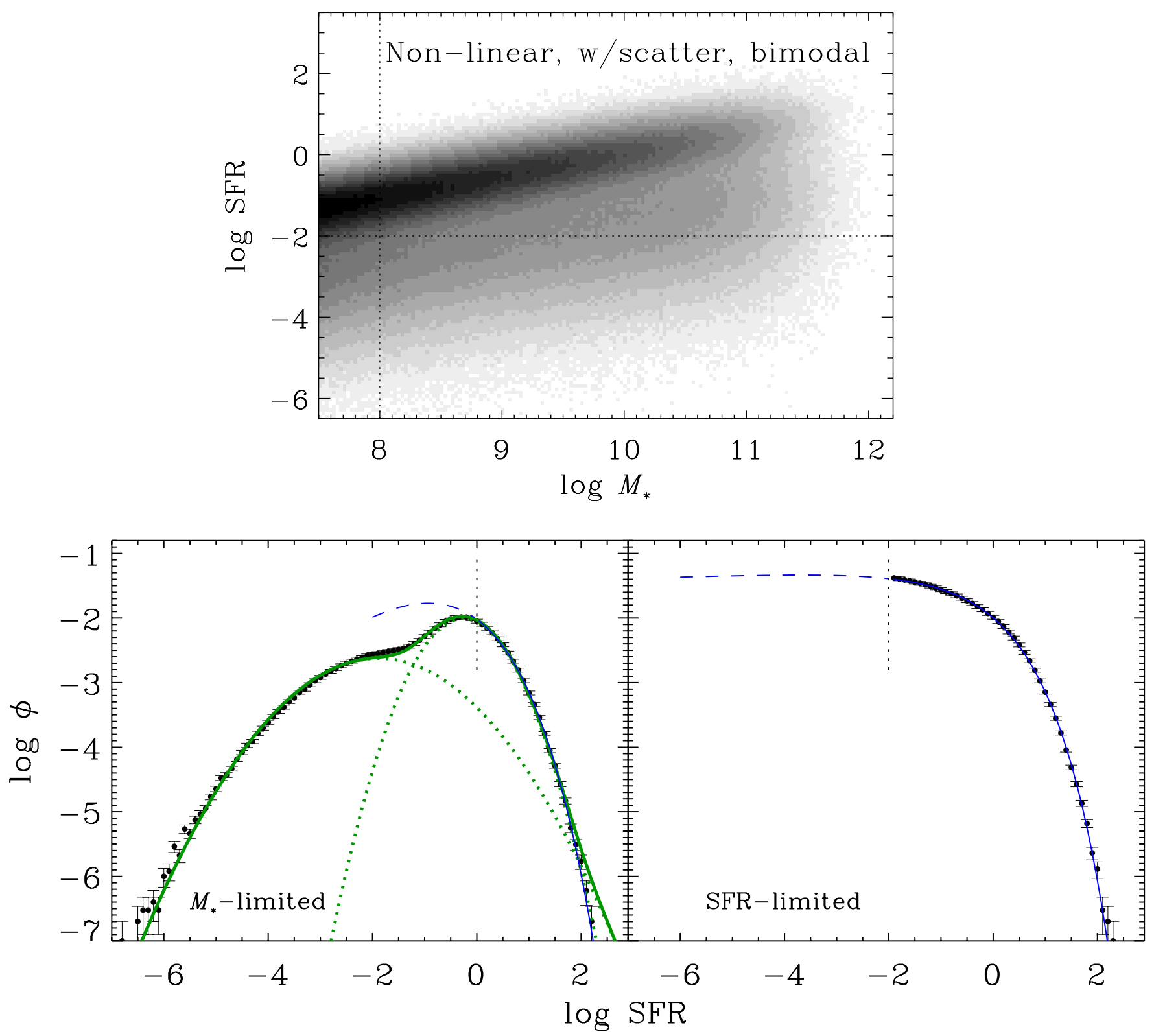

Figure 3. Relation 3 and the resulting SFR functions. SFRs are bimodal and include the scatter around a star-forming and a passive sequence, with the fraction of galaxies in each sequence depending on mass as explained in Section 2.4. Upper panel shows the mock SFR-mass relation. SFRs on the passive sequence reach very low values. Dotted lines show limits of mass- and SFR-limited samples. Lower panels show the resulting SFR functions for the mass-limited (left, log $M_{*}>8$ ) and the SFR-limited case (right, $\log$ SFR $>-2$ ). Vertical dashed lines indicate the lower range used in fitting the extended Schechter functions (thin, blue curves). Extended Schechter functions and Saunders functions (not shown) yield good fits in the fitted regions. For the mass-limited case we also fit a double Gaussian (in log SFR, thick green curve), which produces a good fit for the entire SFRF.

(A color version of this figure is available in the online journal.)

mass-SFR dependence in the simple unimodal case with no scatter. As a result of that, the exponential tail changed into a Sérsic function. Now, we consider another alternative: a function that like Schechter and extended Schechter maintains the power law at the low end, but now features a Gaussian (in log SFR) at the high end. We refer to it as the Saunders function, since it was first proposed in Saunders et al. (1990) to model the $\mathrm{LF}$ at $60 \mu \mathrm{m}$. The linear form of the Saunders function can be expressed as

$$
\Phi_{\mathrm{S} 90}(X) d X=\frac{\phi^{\star}}{X^{\star}}\left(\frac{X}{X^{\star}}\right)^{\gamma} \exp \left(-\frac{\log ^{2}\left(1+X / X^{\star}\right)}{2 \sigma^{2}}\right) d X .
$$

Note that the "Gaussian" part of this function tends to a Gaussian when $X>X^{\star}$ and to a constant when $X<X^{\star}$. This modification (the addition of 1 in the argument of $\log$ ) allows the low end to transition smoothly into a power law.

Fitting the Saunders function to an SFRF constructed using the bimodal SFR-mass relation and SFR-limited sample (Figure 3 (lower right)), we obtain a very good fit with $\chi^{2}=0.5$. This is slightly worse than what the extended Schechter fit yielded $\left(\chi^{2}=0.3\right)$; however, whether one or the other is better will depend on the level of scatter, especially in the SF sequence. We know that for the unimodal SFRs, in the limit of zero scatter the extended Schechter function is a perfect analytic description, but this will be less true for large scatter. For the scatter 
assumed here ( 0.4 dex for the SF sequence), both functions are basically equally good approximations.

The Saunders function has one significant advantage over the extended Schechter function: its parameters are less easily perturbed by errors in SFRF and, therefore, the relative accuracy of the parameters corresponding to the Saunders fit is higher. In the case of the bimodal SFRF discussed here the Saunders fit has $\sim 2.5 \times$ smaller errors in $\log \mathrm{SFR}^{\star}$ and $\log \phi^{\star}$ and $5 \times$ smaller uncertainty in the "faint"-end slope. This is the result of the significantly lower level of covariance among the parameters of the Saunders function. On average the Pearson correlation index for Saunders function parameters is 0.70 , while it is 0.94 for extended Schechter. Most importantly, the parameters describing the "faint" and the "bright" ends have a correlation of only 0.33 in the Saunders function $(\gamma$ and $\sigma)$ and yet 0.87 in the extended Schechter function $\left(\alpha^{\prime}\right.$ and $\beta$ ).

While the Saunders function has been proposed for describing the $60 \mu \mathrm{m} \mathrm{LF}$, which can be considered a type of SFRF, the motivation for its introduction was simply to provide a functional form that better describes the real data than the standard Schechter function. Here, we show that the reasons behind such good representation have to do with the nature of SFR and its relation to the stellar mass.

Turning now to the mass-limited SFRF (Figure 3 (lower left panel)), if we only aim to fit the part to the right of the turnover $(\log S F R \geqslant 0)$, then the same conclusions hold as in the SFR-limited case: the extended Schechter function represents an excellent fit $\left(\chi_{r}^{2}=0.5\right.$; blue line in Figure 3 (lower left panel)). Similarly well does the Saunders function (not shown). Is it possible to successfully fit the entire distribution including the low-end drop and the dip? Both the extended Schechter function and the Saunders function are monotonic and feature a power law at the low end, so they will be incapable to reproduce the inflection or the low-end drop. Thus, we test a new functional form: a double (i.e., composite) Gaussian function (in log SFR), in which each Gaussian should fit one of the two modes of SFR distribution. This function is given in the log form as

$$
\Phi_{\mathrm{GG}}(\log X) d(\log X)=\left(\phi^{\star}{ }_{P} G_{P}+\phi^{\star}{ }_{S} G_{S}\right) d(\log X)
$$

where

$$
G_{P}=\frac{1}{\sigma_{P} \sqrt{2 \pi}} \exp \left(-\frac{\log ^{2}\left(X / X_{P}^{\star}\right)}{2 \sigma_{P}^{2}}\right)
$$

represents the Gaussian corresponding to the passive population and

$$
G_{S}=\frac{1}{\sigma_{S} \sqrt{2 \pi}} \exp \left(-\frac{\log ^{2}\left(X / X_{S}^{\star}\right)}{2 \sigma_{S}^{2}}\right)
$$

the Gaussian of the SF population, each with its own standard deviation and peak position. Double Gaussian features six parameters, but the covariances are weak between each set of three.

Indeed, when we fit the double Gaussian, we obtain very good results (solid green line in Figure 3 (lower left panel), with the individual components shown with dotted green lines; on a log-log plot the double Gaussian is represented as two parabolas). The fitted Gaussian standard deviations are $\sigma_{P}=1.0$ and $\sigma_{S}=0.5$, reflecting the scatters of the passive and $\mathrm{SF}$ sequences. As in the case of the Saunders function, the parameters of the double Gaussian are significantly less sensitive to SFRF uncertainties than the parameters of the extended Schechter function. While much better than any other practical alternative, the double Gaussian is not a perfect fit $\left(\chi_{r}^{2}=1.9\right)$.
It produces slightly stronger inflection (deeper dip) than what is seen in the SFRF. Also, recall that when we constructed SFRs we required that SFRs from the passive sequence do not exceed SFRs of the SF sequence by more than 0.4 dex. However, no such restriction is imposed in the fitting, so the passive Gaussian extends a bit too much at the high end. Clipping the passive Gaussian where it starts to exceed the SF Gaussian brings the reduced $\chi_{r}^{2}$ to 1.5 .

Note that the success of the double Gaussian in fitting the SFRF is not a mere consequence of the fact that in each mass bin we modeled SFR-mass relations as the sum of two Gaussians because the slope of the SFR-mass relation is significantly larger than zero (i.e., the Gaussians from different mass bins have different centers in SFR).

To our knowledge the double Gaussian was not previously considered as a functional form for the SFR function. A single Gaussian (lognormal function in linear SFR) has been suggested by Martin et al. (2005). However, such a form (a parabola on the $\log -\log$ plot) is apparently inadequate when the details of the SFR function are considered, i.e., when it is measured precisely below $\log$ SFR $\lesssim 0$. Fontanot et al. (2012) note the "double peak" feature in their $0.4<z<1.8$ mass-limited SFR functions and suggest that this "peculiar feature" might be connected to bimodality. Our analysis shows that bimodality is indeed the explanation.

\subsection{Summary: Functional Forms Describing SFR Function}

Summarizing the modeling section, we conclude that the Schechter function is an inadequate description for any realistic SFR function. Instead, SFRFs derived from the SFR-limited sample can be sufficiently well modeled using either the extended Schechter function or the Saunders function (a power law with a Gaussian decline at the high end). The latter is recommended because its parameters, being less covariant, will be more stable and yield higher relative accuracy. For the masslimited SFRFs the double Gaussian represents an excellent solution. Note that in all of these cases the fitted parameters will not directly be interpretable as the parameters of the underlying MF or the SFR-mass relation, but they will provide robust descriptions of SFRFs that can be compared from one study to another.

\section{STAR FORMATION RATE DENSITY}

There are two primary reasons for which describing the SFR function with an analytic function form is useful. One is to characterize this distribution through parameterization, to facilitate the study of its evolution with redshift. The second is to use the parameterization to infer, by integration that involves extrapolation, the total SFR density. In that case the Schechter formulation is especially practical because its cumulative distribution function is finite when $X \rightarrow 0$ and has a simple analytic expression. Since, as we have demonstrated, the Schechter formulation does not provide an adequate description of the observed SFR function, alternative methods are needed for inferring the total SFR density. While all of the alternative functions that we have considered (the extended Schechter, Saunders, and the double Gaussian) also have finite cumulative distributions, they cannot be integrated to yield functions in a closed form. Therefore, we will instead employ our bimodal model (constructed based on $z \sim 0.1$ SFRs) to provide numerical correction coefficients to be applied to the SFR density determinations in the local universe obtained from the 


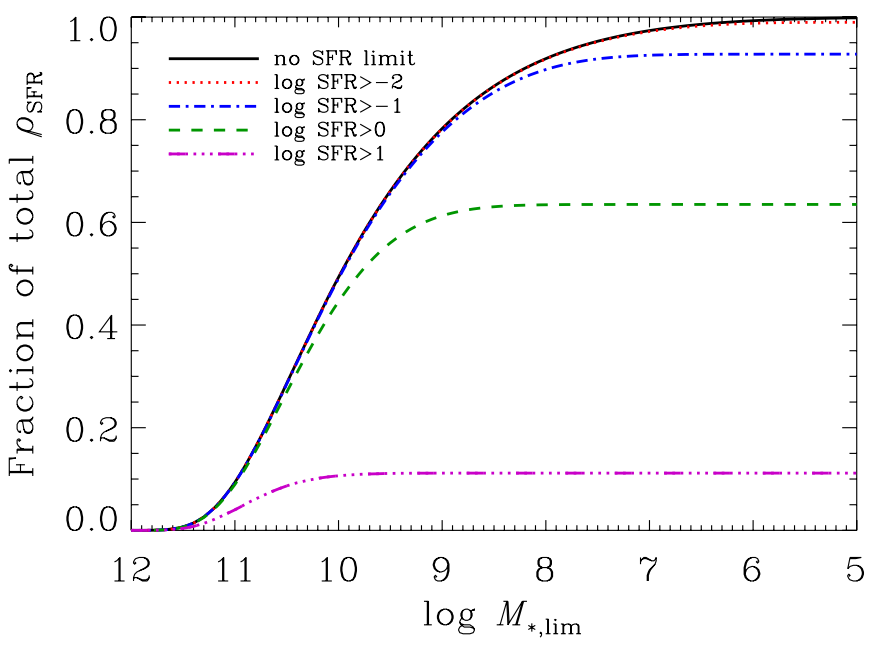

Figure 4. Fraction of the total SFR density that is accounted for by integrating mock bimodal SFRF down to a given mass limit ( $x$-axis) and SFR limit (different curves). To probe $90 \%$ of the SFR density in the local universe requires sampling galaxies to $\log M_{*} \approx 8$ and $\log$ SFR $>-1$. The figure can be used to determine the correction factors ( 1 /fraction) to be applied to the SFR density determinations in the local universe $(z \sim 0.1)$ obtained from the direct numerical integration of the SFRF and to estimate the yield of planned SFR surveys.

(A color version of this figure is available in the online journal.)

direct numerical integration of the observed SFRF down to some SFR limit, based on a sample with some mass limit.

The fraction of the total SFR density that will be present in the observed local SFRF to a given limit is shown in Figure 4. The mass limit can be read continuously from the $x$-axis, while each of the curves represents some SFR limit. The black solid curve shows the fraction accounted for at different mass limits if there was no SFR limit. Going to $\log M_{*}=8$ in such a case (corresponding to the mass limit that we explored heretofore) probes $92 \%$ of the local SFR density; i.e., the values obtained from integrating the SFRF in Figure 3 (lower left panel) would have to be corrected upward by $9 \%$. It should be noted that in the presence of a mass limit the correction is not due to mere extrapolation of the SFRF but serves to correct for lower-mass galaxies that contribute at a range of SFRs. Having the SFR limit of $\log \mathrm{SFR}=-2$ (red dotted curve) leads to negligible difference with respect to the case with no SFR limit. On the other hand, a limit of $\log$ SFR $=0$ (green dashed curve) captures only $64 \%$ of the SFR density in the local universe.

We emphasize that if only the SFR limit is present, one could recover the missing SFR density by simply extrapolating the faint-end power-law tail (of course, assuming that the slope is well constrained), but if the mass limit is also present, one would have to account for it too, using, for example, the provided figure or performing modeling similar to that presented here. ${ }^{11}$ A similar technique can also be used to estimate the yield of a planned survey or to estimate corrections due to incompleteness.

\section{THE OBSERVED LOCAL SFR FUNCTION}

Next we investigate the shape of the observed SFR functions and test whether they can indeed be described with the functional

\footnotetext{
11 At http://www.astro.indiana.edu/ salims/sfrf we provide an IDL script, based on the bimodal relation, for calculating the fraction of SFR density recovered to a given mass and SFR limits and for a given redshift.
}
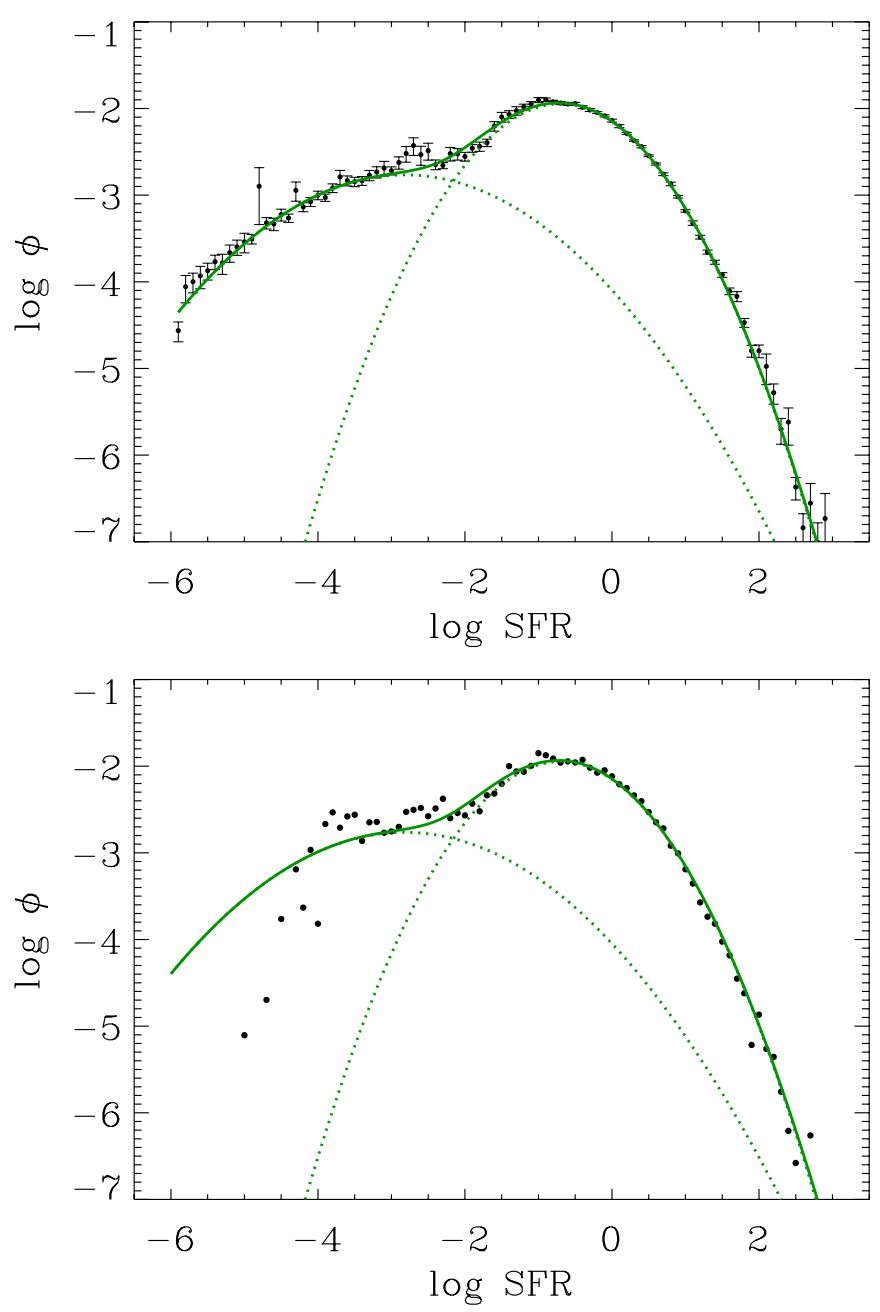

Figure 5. Upper panel: the observed $z \sim 0.1$ SFR function obtained from Salim et al. (2007) data, where SFRs where obtained from UV and optical SED fitting of an optically selected sample with $\log M_{*}>8$. SFRF is a composite of each galaxy's SFR probability distribution, not a single value. Error bars are determined from the standard deviation of bootstrap samples. Green curves represent double Gaussian fits. Lower panel: same as upper panel except that the SFRF was constructed such that each galaxy's SFR was given by a single value (mean of the probability distribution). Green curves are repeated from the upper panel. Most of the SFRF stays unchanged.

(A color version of this figure is available in the online journal.)

forms determined based on simulated SFRs in Section 2. We construct the SFR function from UV/optical-based SFRs of $\approx 50,000$ galaxies from Salim et al. (2007). This data set only has a mass limit $\left(\log M_{*}=8\right)$ and can formally yield specific SFRs as low as $\log \left(\mathrm{SFR} / M_{*}\right)=-12$, which correspond to very low SFRs even for massive galaxies. The SFRF constructed from these data is shown in Figure 5 (upper panel). As expected for the mass-limited sample, the SFRF drops off at low values. Overall, its shape is quite similar to the bimodal mock SFRF presented in Figure 3 (lower left panel). This is not too surprising because the construction of the mock SFRs was guided by these observations, but it should be kept in mind that the SFR-mass relations we used incorporated some simplifying assumptions (e.g., fixed scatter). We previously concluded that the double Gaussian function is the most suitable description of the mock bimodal SFRF with a mass limit. We fit that function to the observed data points and indeed obtain a very good fit (shown by green curves in Figure 5 (upper panel)). The resulting goodness 
of fit is $\chi_{r}^{2}=1.7$. The parameters of the best fit are

$$
\begin{aligned}
& \log \mathrm{SFR}_{P}^{\star}=-2.82, \quad \sigma_{P}=1.14, \quad \log \phi_{P}^{\star}=-2.31 \\
& \log \mathrm{SFR}_{S}^{\star}=-0.70, \quad \sigma_{S}=0.72, \quad \log \phi_{P}^{\star}=-1.69 .
\end{aligned}
$$

To construct the SFRF, we co-added $1 / V_{\max }$-weighted probability distribution functions for each galaxy's SFR. This is possible because the SFRs in Salim et al. (2007) were obtained using the Bayesian SED fitting. In Bayesian SED fitting each model SED contributes (proportionally to $e^{-\chi^{2} / 2}$ ) to the probability distribution of a galaxy parameter (such as the SFR). Using full probability distributions for each galaxy's SFR (instead of a singular value) has the advantage that it produces more realistic distributions for an ensemble of galaxies. Since full probability distributions of SFRs are not always available, we also considered the SFRF where each galaxy is represented by a single value of SFR (the mean of the probability distribution). Such an SFRF is shown in Figure 5 (lower panel). We overlay it with the best double Gaussian fit obtained using full probability distributions. There is an excellent agreement between the two especially in the region fit by the star-forming Gaussian, including the high-end tail. The latter means that the distribution in the high-end tail is not due to some galaxies having broad SFR probability distributions reaching very high values. Discrepancies start to appear only below $\log \mathrm{SFR} \lesssim-3$, because galaxies with very low SFRs usually have broad (poorly constrained) probability distributions, so when these are collapsed into a single value for SFR, their extent toward very low values gets somewhat compressed. In any case, such low SFRs have very little effect on any global characterization of the SF.

Our mock SFR functions in Section 2 assumed that masses are drawn from a Schechter function. Deviations of the MF from the Schechter form are known (Baldry et al. 2008), but they are relatively small and mostly pertain to lower-mass passive galaxies (Peng et al. 2010). The ability to reproduce the observed SFRF using functions suggested by mock SFRFs confirms that these deviations are not significant in the context of this study.

\section{DISCUSSION}

Our analysis of mock SFRFs and observations from Salim et al. (2007) show that real SFRFs have significant departures with respect to a Schechter function. One usually determines the SFR based on the luminosity of a tracer population of young stars, such as the far-UV (FUV) continuum luminosity, emission line luminosity (e.g., $\mathrm{H} \alpha$, O II, or $\mathrm{Pa} \alpha$ ), or the dust luminosity in some part of the IR SED (polycyclic aromatic hydrocarbon (PAH) lines, mid-IR continuum, far-IR continuum, or the total IR luminosity). It is then to be expected that the $L F s$ of these various tracers would show similar departures from the Schechter distribution as the SFRF. While such departures have been known for a long time in the IR (especially the far-IR, e.g., Lawrence et al. 1986; Saunders et al. 1990; Takeuchi et al. 2003), there is a general consensus that the FUV LF and the H $\alpha$ LF $d o$ to a large degree agree with the Schechter function (e.g., Wyder et al. 2005 for UV and Gallego et al. 1995; Ly et al. 2011 for $\mathrm{H} \alpha$ ). How can we explain this apparent inconsistency? As the analysis in this section will show, the primary reason for this is because the observed, uncorrected $\mathrm{UV}$ and $\mathrm{H} \alpha \mathrm{LFs}$ have a form that is similar to a Schechter function by coincidence (similarly for LFs where dust is "corrected" by applying average statistical relations). On the other hand, LFs where the luminosity of each galaxy is individually dust corrected (thus becoming a true SFR) do show large departures from the Schechter form in line with our analysis for SFRFs. The departure of the LFs from the Schechter form, even with individually applied dust corrections, is more difficult to recognize in high-redshift studies that feature small samples with the resulting LFs having limited dynamic ranges, which is why this section will discuss more robust observational evidence from lower redshifts.

\subsection{UV LFs}

The characterization of galaxies in the UV has greatly improved with the launch of GALEX, which surveyed most of the sky in two UV bands (FUV, $1500 \AA$; near-UV (NUV), $2300 \AA$ ). Based on the GALEX/2dF observations of $\sim 1000$ galaxies, Wyder et al. (2005) presented FUV and NUV LFs for local $(z<0.1)$ galaxies. These "early" UV LFs were satisfactorily fit with Schechter functions in line with previous UV studies. In Figure 6 (upper panel), we present an updated version of the FUV LF based on $\sim 30,000$ galaxies from Salim et al. (2007). All magnitudes are on the $\mathrm{AB}$ system and are $K$-corrected to $z=0$ and corrected for Galactic reddening. Open points show the LF of FUV absolute magnitude not corrected for internal dust attenuation, i.e., like those of Wyder et al. (2005) and many other works that present uncorrected UV LFs. A standard Schechter function is fit to the uncorrected LF (red curve). While it visually appears as a good fit, the large sample reveals that $\chi_{r}^{2}$ is 3.7 , a relatively large value. A closer look reveals that the faint-end slope of the fit is slightly steeper than the LF points and also that the fit falls somewhat more steeply at the bright end ( $\left.M_{\mathrm{FUV}} \lesssim-20\right)$. Wyder et al. (2005) were unable to identify these discrepancies because their LF had significantly larger error bars and, moreover, because the brightest point of their LF was at $M_{\mathrm{FUV}}=-20$, just before the departure from the Schechter form starts to become apparent at the bright end. Indeed, Schiminovich et al. (2007), using the same data set as the one we use, but analyzed independently, mention the high-end deviation from Schechter. They tentatively ascribed it to the AGN contamination in the UV. However, broad-line AGNs that could affect the UV continuum were already removed in these samples, so this explanation seems unlikely. In any case, this not-so-perfect agreement between the UV LF and Schechter function was generally neglected.

Altogether, the observed UV LF has qualitatively small departures from the Schechter form, while given our results in Sections 2 and 4 we would expect an LF of SFR type (such as the UV LF) to be very poorly fit by the Schechter function. This is because the UV LF, not corrected for dust, is not equivalent to an SFRF. Indeed, the departure from the Schechter form becomes much more severe $\left(\chi_{r}^{2}=24\right)$ when the UV LF is constructed from dust-corrected FUV absolute magnitude, which we show as solid dots in Figure 6 (upper panel). The dust attenuation applied in Figure 6 is obtained on a galaxy-by-galaxy basis from fitting of the full UV-optical SED (Salim et al. 2007) and is mostly constrained by the UV slope. If, on the other hand, one was to apply a fixed dust correction, such a "corrected" LF would simply be the uncorrected LF shifted to brighter magnitudes, while the shape and the steep, Schechter-like bright end would remain. Interestingly, even applying somewhat more sophisticated statistical dust correction brings the "corrected" LF only slightly closer to its true shape. For example, many studies apply a mass- or an optical-luminosity-dependent dust correction. From our data on SDSS/GALEX SF galaxies we find that the medians in mass bin yield the following relation 

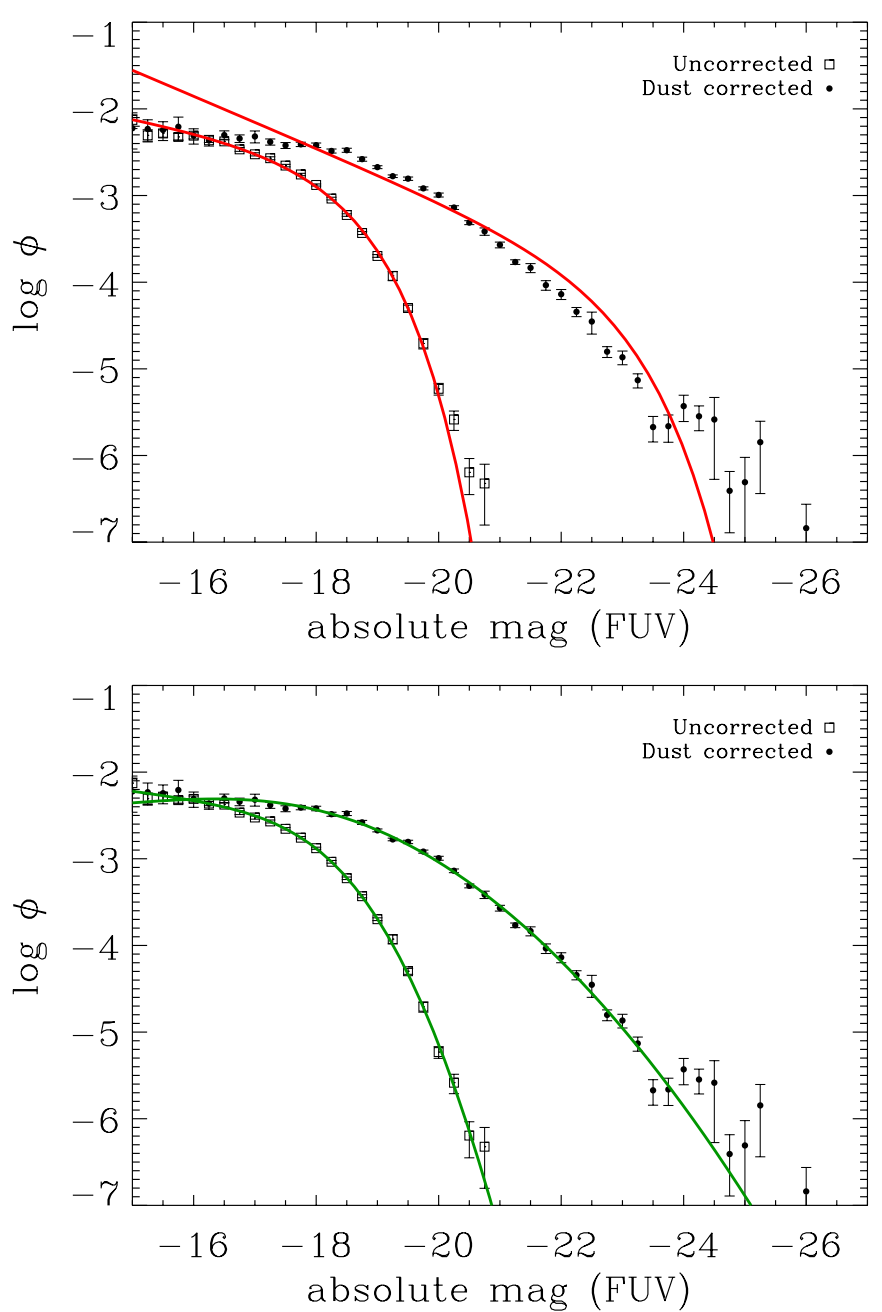

Figure 6. Upper panel: far-UV luminosity functions based on the Salim et al. (2007) sample with (dots) and without (open squares) dust correction. Dust corrections are determined on a galaxy-by-galaxy basis. Error bars are determined from the standard deviation of bootstrap samples. Red lines represent best-fitting Schechter functions. A Schechter function is an acceptable fit only for the uncorrected LF. Lower panel: LFs are repeated from the upper panel, but the fitting functions (green curves) are now Saunders et al. (1990) functions, which feature Gaussian high ends instead of exponentials in the Schechter function. They provide excellent fits for both the uncorrected and the corrected LFs. An LF corrected using average statistical relations (e.g., between dust attenuation and stellar mass) would also yield steep Schechter-like bright-end slopes, and not the much shallower slope of the true SFRF.

(A color version of this figure is available in the online journal.)

between FUV attenuation and stellar mass:

$$
A_{\mathrm{FUV}}=0.71 \log M_{*}-5.16 \text {. }
$$

If we apply this statistical correction to FUV absolute magnitudes, the resulting LFs still feature a relatively steep bright end, which in LFs with smaller dynamic range could again easily be misinterpreted as conforming to a Schechter function. The reason why even the mass-dependent dust correction cannot reproduce the true SFRF is because at any given mass the dispersion in dust corrections is very large (we find 0.7 mag scatter in $A_{\mathrm{FUV}}$ at $\log M_{*}=10.5$; similarly large scatter can be seen in the Garn \& Best 2010 analysis of SDSS Balmer decrements; their Figure 4), and it is the values that scatter above the average relation that are important in shaping the bright end of the LF.
Guided by our previous considerations regarding the SFRF, we can attempt to fit more appropriate functions to both the uncorrected FUV LF (where the departures from Schechter are relatively small, but not negligible) and the dust-corrected FUV LF (where departures are severe). Since the GALEX sample is similar to an SFR-limited sample as it is $M_{\mathrm{FUV}}$ limited, we try both the extended Schechter function (=power law + Sérsic) and the Saunders function (=power law + Gaussian). We find that both functions represent good fits to both the uncorrected and the dust-corrected FUV LFs, but that the Saunders function is somewhat better $\left(\chi_{r}^{2}\right.$ of 0.5 and 1.9 for the uncorrected and corrected LFs, respectively, versus $\chi_{r}^{2}$ of 0.8 and 4.5 for the extended Schechter fit). We show the best Saunders fits as green curves in Figure 6 (lower panel). Note that the high-end tail of the uncorrected LF is now well fit. The parameters of the best Saunders fit are

$$
M_{\mathrm{FUV}}^{\star}=-17.49, \quad \sigma=0.31, \quad \gamma=-1.23, \quad \log \phi^{\star}=-2.41
$$

for the uncorrected FUV LF and

$M_{\mathrm{FUV}}^{\star}=-16.05, \quad \sigma=0.73, \quad \gamma=-0.83, \quad \log \phi^{\star}=-2.24$

for the dust-corrected one.

We now return to the apparent puzzle of why the uncorrected FUV LF is reasonably well fit with a Schechter function (left red curve in Figure 6 (upper panel)) when our analysis has shown that the Schechter function should be an appropriate description for quantities that are proportional to the mass and have small scatter with respect to it (for example, the optical or the near-IR luminosity), which UV luminosity is not. Here we show that this near match is a coincidence arising from two effects that approximately cancel out: sub-linearity of the FUV luminosity-mass relation and the scatter in that relation. The dust correction is on average larger in more massive star-forming galaxies (e.g., Wang \& Heckman 1996; Garn \& Best 2010); therefore, the slope between FUV luminosity not corrected for dust and the stellar mass will be even lower (less linear) than between the SFR (i.e., dust-corrected FUV luminosity) and mass. Indeed, we determine this slope to be $\beta=0.32$ (while it was $\beta=0.65$ for SFR, i.e., dust-corrected FUV luminosity, Equation (1)). Based on this fact alone, the Schechter function should be expected to be an even worse description for the uncorrected FUV LF. We show this in Figure 7. The dashed line shows how the LF would look if FUV luminosity was linearly related to mass, without any scatter. This is simply a Schechter function. However, the actual dependency is sub-linear with $\beta=0.32$. Such an LF, but still with no scatter in $L$ (FUV) versus mass, is shown as a dotted curve. It is much steeper at the bright end than the Schechter function, and the high-end tail is shifted to the left. However, we also need to take into account the scatter of $L$ (FUV) at a given mass. Therefore, we convolve the dotted curve with a Gaussian with $\sigma=0.65 \mathrm{mag}$ to obtain the final LF (solid line). Convolution has the effect of making the tail shallower again, which coincidentally yields a distribution that resembles the Schechter function. The differences are relatively subtle (the slightly more shallow high-end tail and somewhat less steep faint-end slope) and will be easily masked in LFs based on up to a few thousand galaxies. As we have seen, these differences can be revealed with LFs constructed from order-ofmagnitude larger samples (Figure 6 (upper panel)).

This approximate cancellation of sub-linearity and scatter happens to hold only for uncorrected luminosity. Since the dustcorrected FUV luminosity is less sub-linear with respect to the 


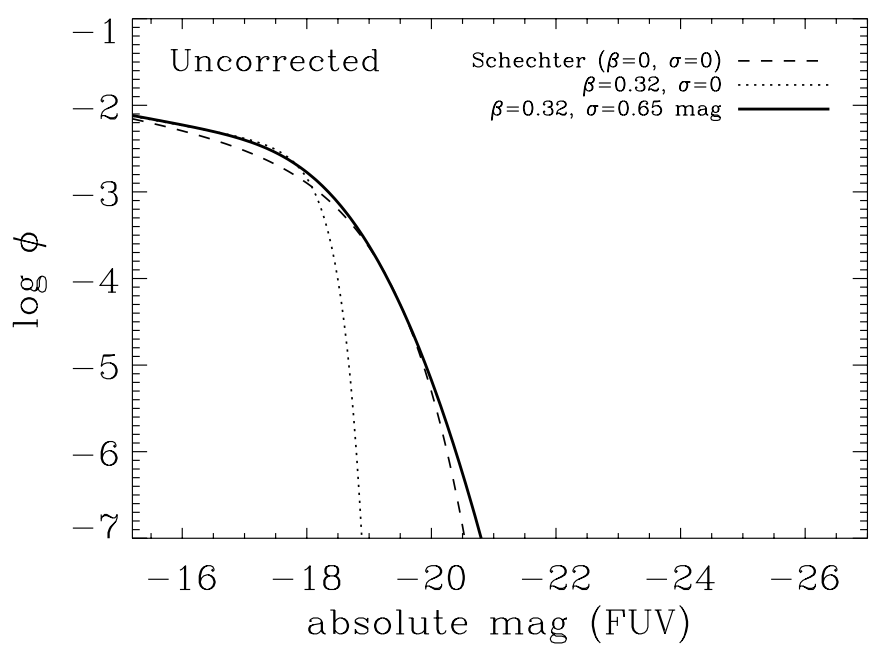

Figure 7. Schematic explanation as to why the Schechter function appears to be an adequate description of the uncorrected FUV LF despite the very different character of UV and optical/near-IR populations. For a distribution to have a Schechter form it needs to have a linear dependence on mass with no scatter (dashed curve). Severe sub-linearity, as in FUV luminosity vs. mass, makes the distribution much steeper (dotted curve), but adding the right amount of scatter to such a nonlinear relation (solid curve) modifies the high-end tail into a form that resembles the Schechter function (dashed curve). Similar principles would apply to LFs in near-UV or the H $\alpha$ LF.

stellar mass, the dotted line shifts less to the left. When scatter is added to it, it moves the LF more to the right and with a shallower slope than that of the Schechter function (Figure 8), yielding what can be considered a true SFRF and which is well described by the Saunders function.

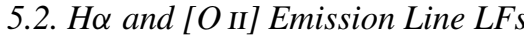

All of the considerations laid out regarding the UV LFs are also applicable to $\mathrm{H} \alpha$ LFs. This is because the $\mathrm{H} \alpha$ luminosities also require significant dust corrections to be representative of the true SFRs. Ultimately, the uncorrected $\mathrm{H} \alpha \mathrm{LF}$ is again only approximately and coincidentally described by a Schechter function. Accurate $\mathrm{H} \alpha \mathrm{LFs}$ do show deviations. This has been noted by Gilbank et al. (2010) for both $\mathrm{H} \alpha$ and [O II] LFs from SDSS spectra $(z \sim 0.1)$ and by Zhu et al. (2009) in their $z \sim 1$ [O II] LF from DEEP2 spectra. These studies instead fit a double power law (broken line on a log-log plot) to the observed emission-line LFs. The dust-corrected LFs would show even stronger departures from the Schechter form if the dust attenuation is determined for each galaxy individually. In either case, a function such as the Saunders or the extended Schechter would provide an optimal description.

$\mathrm{Pa} \alpha$ is emitted in the near-IR, so it requires very little dust correction (Calzetti et al. 2007). We anticipate that once Pa $\alpha$ LFs are constructed, they will depart significantly from the Schechter form and will feature a Gaussian or a Sérsic high-end tail instead.

\subsection{IR LFS}

The inadequacy of the Schechter formulation for describing IR LFs was noticed already in the mid-1980s based on IRAS $60 \mu \mathrm{m}$ data. Thus, Lawrence et al. (1986) proposed a double power-law fit (i.e., the exponential cutoff of the Schechter function was replaced by a less steep power law), while Saunders et al. (1990), using an order-of-magnitude larger sample (2800 versus 300), noticed the curved high-end tail of the $60 \mu \mathrm{m} \mathrm{LF}$ and so instead recommended the combination of a power law for the low end and the Gaussian for the high end, which

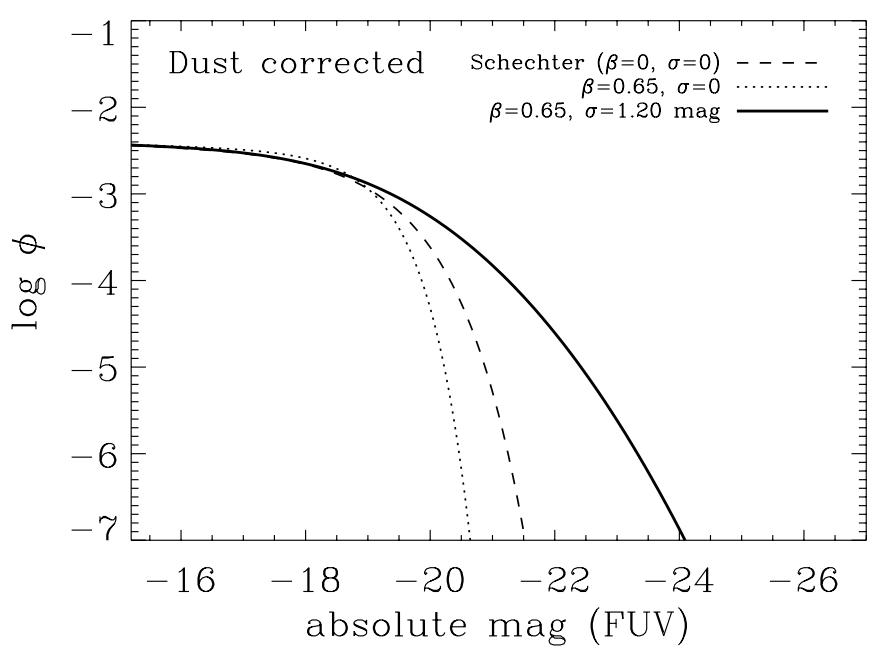

Figure 8. Schematic explanation as to why the Schechter function is not an adequate description of the dust-corrected FUV LF (i.e., SFRF). The mean relation between luminosity and mass is closer to linear, leading to a smaller shift to the left (dotted curve), which results in a flatter LF when scatter is applied (solid curve) and a much larger departure with respect to the Schechter function (dashed curve).

we refer to as the Saunders function (Equation (8)). ${ }^{12}$ The Saunders function remains an excellent functional form for the LFs based on the most recent reductions of the IRAS $60 \mu \mathrm{m}$ data, containing five times as many galaxies as used in Saunders et al. (1990) and spanning eight orders of magnitude in space density (Takeuchi et al. 2003; see also Wang \& Rowan-Robinson 2010). Despite the success of the Saunders function, some studies continue to use the simpler double power law (with or without a soft transition between the two power laws) to model the far-IR LF, especially when smaller sample sizes $\left(\lesssim 10^{3}\right)$ that probe a smaller dynamic range of the LF are involved (e.g., Sanders et al. 2003), or when the LFs are constructed for luminosities at $\lambda>60 \mu \mathrm{m}$, where the measurements typically have lower accuracy (e.g., Goto et al. 2011). The two forms can be rather similar, and distinguishing between them requires very accurate LFs.

The situation appears to be similar for the mid-IR LFs. Shupe et al. (1998) find that the $25 \mu \mathrm{m}$ LF constructed from IRAS data has a shallow high-end tail and can be modeled as a double power law. Using Infrared Space Observatory (ISO) $15 \mu \mathrm{m}$ measurements, Xu (2000) and Pozzi et al. (2004) also obtained LFs with shallow high-end tails that would be incompatible with a Schechter distribution. Given these results, it comes as a surprise that Huang et al. (2007) found that the high end of the $8 \mu \mathrm{m} \mathrm{LF}$ is rather steep and is well fit by a Schechter function. They find that this is the case even after they subtract the stellar continuum contribution, i.e., when the resulting $8 \mu \mathrm{m}$ emission comes primarily from PAH line emission, which is considered to be a tracer of young populations and therefore of SF (Förster Schreiber et al. 2004; Díaz-Santos et al. 2008). Huang et al. (2007) explicitly point out that their results are in contrast with the Saunders et al. (1990) $60 \mu \mathrm{m}$ LF that has excess counts above the Schechter function. They also point out that this agreement with the Schechter function cannot be explained by their removal of galaxies harboring AGNs. Namely,

\footnotetext{
12 A number of papers published since 2005 refer to the Saunders function as the double exponential, which we find inaccurate and confusing. In mathematics the double exponential refers to functions of the form $f(x)=a^{b^{x}}$, which the Saunders function is not.
} 
dust-obscured AGNs could heat the surrounding dust to relatively high temperatures, resulting in the IR SED component that peaks in the mid-IR (Fu et al. 2010). Huang et al. (2007) offer no explanation for the puzzling inconsistency between their $8 \mu \mathrm{m}$ PAH LF and LFs at longer IR wavelengths. We suggest that the reason behind this is because the PAH emission is perhaps not a good tracer of the current SF. Namely, there are indications that the PAHs exist outside of $\mathrm{H}$ II regions where they are heated by the general interstellar radiation field produced by older stars (Calzetti 2011). By tracing intermediate-age ( $\sim 1 \mathrm{Gyr})$ or older stellar populations, PAH luminosity immediately becomes more closely related to the stellar mass and less so to the current SF, and thus the PAH LF can be expected to more closely follow the MF and therefore the Schechter distribution. In other words, the PAH LF is perhaps not a true SFRF.

The curious fact that some LFs of SFR type (primarily UV and $\mathrm{H} \alpha$ LFs) are Schechter-like, which we now explain to be unrelated to the Schechter form of LFs of mass type, and the consequent uncertainties as to the true shape of the SFRF left a vacuum that some recent studies try to fill by proposing that the IR LF function is intrinsically also of the Schechter form, but that some effect not related to SF makes the tail assume a shallower non-Schechter slope. These studies find a culprit among the dust-obscured AGN, which, as mentioned, can affect the IR SED, especially in the mid-IR. If correct, such an explanation would disagree with our conclusion (and the view of many previous IR studies, e.g., Takeuchi et al. 2003, 2010; Buat et al. 2007, 2009) that the IR LF, to the extent that it measures current $\mathrm{SF}$, and correspondingly the SFRFs are intrinsically not of the Schechter form.

We first discuss the evidence for AGN contamination in the mid-IR (where it is expected to be stronger) and then the farIR (which also dominates the total IR luminosity). To test the AGN contamination hypothesis, one needs to remove the AGN contribution to the IR luminosities and construct an SF-only IR LF. Fu et al. (2010) used Spitzer IRS spectra of $z \sim 0.7$ galaxies to construct LFs at 8 and $15 \mu \mathrm{m}$ rest frame. Mid-IR spectroscopy allowed them to decompose SF and AGN components on a galaxy-by-galaxy basis (at least for the galaxies in the bright tail). AGNs were found to dominate at the highest 8 and $15 \mu \mathrm{m}$ luminosities. After removing their contribution, they find that the resulting LFs are well fit by Schechter functions. We note that Fu et al. (2010) probe LFs with good precision over only 1 dex of luminosities, so the shapes of the resulting LFs are not well determined. Following in the footsteps of $\mathrm{Fu}$ et al. (2010), Wu et al. (2011) use mid-IR spectra to decompose starforming and AGN contributions at rest frame 15 and $24 \mu \mathrm{m}$ for a more local sample $(z<0.3)$. They confirm that after correcting for AGN contribution the 15 and $24 \mu \mathrm{m}$ LFs become formally consistent with the Schechter functions; however, we notice that their data do not probe the high end sufficiently well to rule out non-Schechter distribution. ${ }^{13}$ Interestingly, Rujopakarn et al. (2010), who instead of decomposing the AGN contributions completely exclude them, find much smaller difference between the total and SF-only $24 \mu \mathrm{m}$ LFs. Consequently, they fit both with double power laws and do not consider the Schechter function at all. To conclude, there is tentative evidence that after the AGN correction the mid-IR LF is Schechter-like. This possible agreement of the mid-IR LFs with the Schechter

\footnotetext{
13 For example, as shown in their Figure 7, even before the AGN correction their $15 \mu \mathrm{m}$ LF can be fitted by either the Schechter or the Saunders function. Unfortunately, one LF point that could help understand what happens at the high end was omitted from their SF LF in Figure 8(a).
}

distribution could be explained if the mid-IR continuum does not truly trace the current SFR. Indeed, Kelson \& Holden (2010) present a model in which most of the mid-IR emission is produced by carbon asymptotic giant branch stars with ages between 0.2 and 2 Gyr, while Salim et al. (2009) find that the $\sim 15 \mu \mathrm{m}$ luminosity is more tightly correlated with the optical $B$-band luminosity, where intermediate-age stars dominate, than it is with the dust-corrected FUV luminosity of young stars. A significant contribution of low-mass stars in the heating of mid-IR dust would make it more strongly correlated with the cumulative SF and therefore the stellar mass than the current SF. Consequently, the mid-IR LFs may be of MF type and thus be better described by Schechter functions.

AGN contribution can be accessed relatively directly in the mid-IR, where the AGN SED peaks, but it is more uncertain in the far- and the total IR. Wu et al. (2011), extrapolating from their $24 \mu \mathrm{m}$ results, find that the AGN contribution to the total IR luminosity is only $10 \%-20 \%$ (out to $\log L_{\mathrm{IR}}=11.7$ ), yet some studies attempt to correct for AGN contribution in the total IR luminosity. This can be done in two ways. One is to keep all galaxies but remove the fraction of IR luminosity believed to come from an AGN, and the other, more crude method is to remove galaxies showing signs of AGNs from the LF altogether. The IR LF constructed using the former method is equivalent to the SFRF, while the latter will be a lower limit to the true SFRF. The recent example of the IR LF that completely removes galaxies with AGNs is the one presented in Goto et al. (2011), derived from $A K A R I$ data. Goto et al. (2011) show the full IR LF alongside one that removes AGNs based on the number fractions of optically identified AGNs in each IR luminosity bin from Yuan et al. (2010). The Yuan et al. (2010) AGN number fraction is a steeply rising function of IR luminosity. Thus, the Goto et al. (2011) LF of non-AGN galaxies has a steeper bright end than the full LF, but still not so steep to make the authors consider fitting the Schechter function instead of the double power law. We confirm this by performing the fits ourselves on the Goto et al. (2011) LFs. Schechter functions yield a very poor fit for either the total or just the non-AGN LF $\left(\chi_{r}^{2}=10\right.$ and 11, respectively). On the other hand, the Saunders functions produce excellent fits with $\chi_{r}^{2}=0.6$ and 0.7 for the total and non-AGN LFs, respectively. Since the non-AGN LF is only the lower limit to the real SF IR LF, the latter also cannot be a Schechter function. The conclusion that the AGNs are not responsible for the non-Schechter form of the IR LF is also in line with the exquisite LF at $60 \mu \mathrm{m}$ from IRAS data. Takeuchi et al. (2003) show that the $60 \mu \mathrm{m}$ LF does have an excess at $L_{60}>10^{11.5} L_{\odot}$, but this excess is only $\approx 0.2$ dex in $L_{60}$ and lies above the Saunders fit and not just above a putative steep Schechter high-end slope.

Finally, another strong observational confirmation that the true SFRF is not of the Schechter form comes from the LFs of radio galaxies, where the separation between AGNs and the star-forming component can be achieved more easily than in the IR. Thus, for example, Mauch \& Sadler (2007) show that the $1.4 \mathrm{GHz}$ LF of star-forming radio galaxies is very well fit by a Saunders function, but not Schechter.

To summarize, while there seems to be some evidence that the mid-IR LFs, after correcting for the AGN contribution, may be consistent with a Schechter function, we propose that this could be the consequence of the mid-IR luminosity intrinsically tracing SF over longer timescales and therefore being more related to the stellar mass than to the current SF. On the other hand, it appears that for either the far-IR or the total IR 
even very liberal AGN corrections do not produce Schechterlike LFs. On the contrary, far- and total IR LFs remain well described by functions that replace the exponential cutoff of the Schechter function with less steep functional forms, especially the Gaussian (as featured in the Saunders function), in line with the expectations from our simulations.

\subsection{Distribution of Specific SFRS}

Specific SFR (sSFR) normalizes SFR by stellar mass of a galaxy, thus allowing us to asses its SF history and characterize it as bursty, normal, or quiescent. The distribution of specific SFRs (sSFR function) can offer complementary insights to those offered by the SFRF alone. We have already shown that at any given mass the observed SFR distribution is well described by two Gaussians: one for the SF sequence and another for a broad, passive sequence. This double Gaussianity is preserved when the bimodal SFR-mass relation is projected to produce a masslimited SFR distribution. The same is true for the distribution of specific SFRs. It too can be described as the composite of two Gaussians in $\log \left(\mathrm{SFR} / M_{*}\right)$.

Recently, Sargent et al. (2012) have proposed that at $z \sim 2$ what we call the SF sequence and model as a single Gaussian is in itself composed of two Gaussians-one in which the majority of normal SF galaxies lie, and another one forming a bump on the side of the main one, which contains strongly starbursting galaxies. Furthermore, Sargent et al. (2012) present a framework in which this two-mode sSFR distribution extends to lower redshifts, and they show that it can explain the non-Schechter character of the local IR LF. ${ }^{14}$ However, our analysis of the sSFR distribution of 40,000 $z \sim 0.1$ galaxies, performed in exactly the same way, shows absolutely no indication that the SF sequence has a second, starbursting mode. Most likely this mode has become negligible since $z \sim 2$. More importantly in the context of this study is that no second mode in the SF sequence is needed to produce an IR LF (i.e., SFRF) with nonSchechter form. As shown in Section 2.3, the non-Schechter distribution is primarily the result of a scatter in the SFR-mass relation within the unimodal SF sequence alone.

\subsection{Bivariate $(s) S F R-M_{*}$ Distributions}

The approach in this work was to arrive at SFRFs by first modeling the SFR versus $M_{*}$ distribution. The SFRF collapses the information from the bivariate SFR $-M_{*}$ distribution. Therefore, our recommendation is that all studies that report on SFRFs or LFs of SFR type should also construct an SFR (or sSFR) versus $M_{*}$ diagram (even a simple scatter plot) to aid in the interpretation of the SFRF. From such a diagram one should try to determine the slope and the scatter of the SF sequence and get some sense of the fraction of galaxies on the passive sequence, as well as determine the actual mass and SFR limits. For most purposes even crude mass estimates would suffice. Techniques developed for the construction of bivariate LFs (Takeuchi 2010; Takeuchi et al. 2012; Johnston 2011) can also be applied for the construction of formal bivariate SFR $-M_{*}$ and $\mathrm{sSFR}-M_{*}$ distributions.

\section{CONCLUSIONS}

The main conclusions of this study can be summarized as the following:

\footnotetext{
14 To be accurate, Sargent et al. (2012) do not construct an sSFR distribution but an SSFR distribution relative to the peak of the SF sequence. The two are very similar.
}

1. Distributions of the SFR and the stellar mass are fundamentally different. Consequently, the LFs related to mass (optical and near-IR) will differ from dust-corrected LFs related to SF (UV, emission line, IR).

2. SFR distributions (SFR functions) are very poorly described by a Schechter functional form, which is adequate for MFs. Instead, SFR functions (of SFR-limited samples) are very well described by either an extended Schechter function (which replaces the exponential cutoff with a Sérsic function) or a Saunders function (where the high end is described by a Gaussian in $\log$ SFR); see Figures 3 (lower right panel) and 6 (lower panel). Both feature four parameters. In several empirical cases that we tested (our FUV LF and IR LFs of Goto et al. 2011 and Takeuchi et al. 2003), the Saunders function produced somewhat better fits. The Saunders function has an additional advantage that its parameters are less covariant between each other and are therefore more robust to LF measurement errors.

3. SFR functions of mass-limited samples feature a drop at the low end even for volume-complete samples. This drop should not be confused with incompleteness. The shape of SFR functions at the low end is critically sensitive to the presence of mass limits. Mass-limited SFR functions are well described with double Gaussians in log SFR (Figures 3 (lower left panel) and 5).

4. As previous studies have shown, the observed UV (and $\mathrm{H} \alpha$ ) LFs with no dust correction (or with average statistical dust corrections) can approximately be described by a Schechter function (Figure 6 (upper panel)). The Schechter form in them is not fundamental (like it is in optical LFs) but is a consequence of two effects that by chance approximately cancel each other out (Figure 7). Precise LFs (using samples with $>10^{4}$ galaxies) reveal that even the uncorrected UV LF deviates from the Schechter function. UV and $\mathrm{H} \alpha$ LFs need to be dust corrected on a galaxy-by-galaxy basis and need to have at least a moderate dynamic range for departures from the Schechter form to be evident. These requirements are not always fulfilled in high-redshift studies, leading to apparent agreements of UV and $\mathrm{H} \alpha$ LFs with the Schechter form. When properly dust corrected, UV LFs follow our mock SFRFs and are successfully described by Saunders functions.

5. LFs in the far- and total IR, as well as the radio LF for star-forming galaxies, behave like our mock SFRFs and are poorly fit with a Schechter function even when all AGNs are removed. Instead, they are very well fit by Saunders functions.

6. LFs in the mid-IR may intrinsically be Schechter-like (after correcting for the AGN contribution), which could be the consequence of the mid-IR tracing less massive (older) stellar populations; i.e., mid-IR LFs are possibly not real SFR functions but are more closely related to stellar MFs. Therefore, Schechter functions would represent an adequate description.

7. Whenever possible, a bivariate SFR $-M_{*}$ distribution should be considered alongside its projections.

The recognition that optical LFs and MFs can be characterized using a functional form proposed by Schechter (1976) has provided extremely valuable guidance in the study of galaxy populations across a range of redshifts. As our estimates of SFRs have improved in recent years, having a similar tool to apply to SFR distributions will hopefully add to our understanding of galaxies and their evolution. 
I (S.S.) dedicate this paper to the memory of my mother, Mirjana Makra-Salim (1947-2012). I am forever grateful for her selfless love.

\section{REFERENCES}

Baldry, I. K., Driver, S. P., Loveday, J., et al. 2011, MNRAS, 421, 621 Baldry, I. K., Glazebrook, K., \& Driver, S. P. 2008, MNRAS, 388, 945 Baldwin, J. A., Phillips, M. M., \& Terlevich, R. 1981, PASP, 93, 5

Bell, E. F., McIntosh, D. H., Katz, N., \& Weinberg, M. D. 2003, ApJS, 149, 289

Bernardi, M., Shankar, F., Hyde, J. B., et al. 2010, MNRAS, 404, 2087

Binggeli, B., Sandage, A., \& Tammann, G. A. 1988, ARA\&A, 26, 509

Blanton, M. R., Dalcanton, J., Eisenstein, D., et al. 2001, AJ, 121, 2358

Boselli, A., Gavazzi, G., Donas, J., \& Scodeggio, M. 2001, AJ, 121, 753

Bothwell, M. S., Kenicutt, R. C., Johnson, B. D., et al. 2011, MNRAS, 415,1815

Brinchmann, J., Charlot, S., White, S. D. M., et al. 2004, MNRAS, 351, 115

Bruzual, G., \& Charlot, S. 2003, MNRAS, 344, 1000

Buat, V., \& Burgarella, D. 1998, A\&A, 334, 772

Buat, V., Takeuchi, T. T., Burgarella, D., Giovannoli, E., \& Murata, K. L. 2009, A\&A, 507, 693

Buat, V., Takeuchi, T. T., Iglesias-Páramo, J., et al. 2007, ApJS, 173, 404

Calzetti, D. 2011, EAS Publications Series, 46, 133

Calzetti, D., Kennicutt, R. C., Engelbracht, C. W., et al. 2007, ApJ, 666, 870

Cole, S., Norberg, P., Baugh, C. M., et al. 2001, MNRAS, 326, 255

Cortese, L. 2012, A\&A, 543, A132

Dale, D. A., Cohen, S. A., Johnson, L. C., et al. 2009, ApJ, 703, 517

Davé, R., Oppenheimer, B. D., \& Finlator, K. 2011, MNRAS, 415, 11

Díaz-Santos, T., Alonso-Herrero, A., Colina, L., et al. 2008, ApJ, 685, 211

Dutton, A. A., van den Bosch, F. C., \& Dekel, A. 2010, MNRAS, 405, 1690

Elbaz, D., Daddi, E., Le Borgne, D., et al. 2007, A\&A, 468, 33

Felten, J. E. 1977, AJ, 82, 861

Fontanot, F., Cristiani, S., Santini, P., et al. 2012, MNRAS, 421, 241

Förster Schreiber, N. M., Roussel, H., Sauvage, M., \& Charmandaris, V. 2004, A\&A, 419, 501

Fu, H., Yan, L., Scoville, N. Z., et al. 2010, ApJ, 722, 653

Gallego, J., Zamorano, J., Aragon-Salamanca, A., \& Rego, M. 1995, ApJ, $455, \mathrm{~L} 1$

Garn, T., \& Best, P. N. 2010, MNRAS, 409, 421

Gilbank, D. G., Baldry, I. K., Balogh, M. L., Glazebrook, K., \& Bower, R. G. 2010, MNRAS, 405, 2594

Goto, T., Arnouts, S., Malkan, M., et al. 2011, MNRAS, 414, 1903

Hopkins, P. F., Younger, J. D., Hayward, C. C., Narayanan, D., \& Hernquist, L. 2010, MNRAS, 402, 1693

Huang, J.-S., Ashby, M. L. N., Barmby, P., et al. 2007, ApJ, 664, 840

Huang, S., Haynes, M. P., Giovanelli, R., \& Brinchmann, J. 2012, ApJ, 756, 113

Johnson, B. D. 2011, in ASP Conf. Proc. 440, UP2010: Have Observations Revealed a Variable Upper End of the Initial Mass Function?, ed. M. Treyer, T. K. Wyder, J. D. Neill, M. Seibert, \& J. C. Lee (San Francisco CA: ASP), 227
Johnston, R. 2011, A\&A Rev., 19, 41

Kelson, D. D., \& Holden, B. P. 2010, ApJ, 713, L28

Kennicutt, R. C., Jr. 1998, ARA\&A, 36, 189

Kennicutt, R. C., Jr., Lee, J. C., Funes, S. J., et al. 2008, ApJS, 178, 247

Lawrence, A., Walker, D., Rowan-Robinson, M., Leech, K. J., \& Penston, M. V. 1986, MNRAS, 219, 687

Lee, J. C., Gil de Paz, A., Kennicutt, R. C., Jr., et al. 2011, ApJS, 192, 6

Ly, C., Lee, J. C., Dale, D. A., et al. 2011, ApJ, 726, 109

Martin, D. C., Seibert, M., Buat, V., et al. 2005, ApJ, 619, L59

Martin, D. C., Wyder, T. K., Schiminovich, D., et al. 2007, ApJS, 173, 342

Mauch, T., \& Sadler, E. M. 2007, MNRAS, 375, 931

Mobasher, B., Sharples, R. M., \& Ellis, R. S. 1993, MNRAS, 263, 560

Noeske, K. G., Weiner, B. J., Faber, S. M., et al. 2007, ApJ, 660, L43

Panter, B., Heavens, A. F., \& Jimenez, R. 2004, MNRAS, 355, 764

Peng, Y.-j., Lilly, S. J., Kovač, K., et al. 2010, ApJ, 721, 193

Pozzi, F., Gruppioni, C., Oliver, S., et al. 2004, ApJ, 609, 122

Press, W. H., \& Schechter, P. 1974, ApJ, 187, 425

Reddy, N. A., Erb, D. K., Pettini, M., Steidel, C. C., \& Shapley, A. E. 2010, ApJ, 712,1070

Rujopakarn, W., Eisenstein, D. J., Rieke, G. H., et al. 2010, ApJ, 718, 1171

Salim, S., Dickinson, M., Rich, R. M., et al. 2009, ApJ, 700, 161

Salim, S., Rich, R. M., Charlot, S., et al. 2007, ApJS, 173, 267

Sanders, D. B., Mazzarella, J. M., Kim, D.-C., Surace, J. A., \& Soifer, B. T. 2003, AJ, 126, 1607

Sargent, M. T., Béthermin, M., Daddi, E., \& Elbaz, D. 2012, ApJ, 747, L31

Saunders, W., Rowan-Robinson, M., Lawrence, A., et al. 1990, MNRAS, 242,318

Schechter, P. 1976, ApJ, 203, 297

Schiminovich, D., Wyder, T. K., Martin, D. C., et al. 2007, ApJS, 173,315

Sheth, R. K., Bernardi, M., Schechter, P. L., et al. 2003, ApJ, 594, 225

Shupe, D. L., Fang, F., Hacking, P. B., \& Huchra, J. P. 1998, ApJ, 501, 597

Sullivan, M., Treyer, M. A., Ellis, R. S., et al. 2000, MNRAS, 312, 442

Takeuchi, T. T. 2010, MNRAS, 406, 1830

Takeuchi, T. T., Buat, V., \& Burgarella, D. 2005, A\&A, 440, L17

Takeuchi, T. T., Buat, V., Burgarella, D., et al. 2010, in AIP Conf. Ser. 1240, Hunting for the Dark: The Hidden Side of Galaxy Formation, ed. V. P. Debattista \& C. C. Popescu (Melville, NY: AIP), 59

Takeuchi, T. T., Sakurai, A., Yuan, F.-T., Buat, V., \& Burgarella, D. 2012, arXiv: 1206.5950

Takeuchi, T. T., Yoshikawa, K., \& Ishii, T. T. 2000, ApJS, 129, 1

Takeuchi, T. T., Yoshikawa, K., \& Ishii, T. T. 2003, ApJ, 587, L89

Wang, B., \& Heckman, T. M. 1996, ApJ, 457, 645

Wang, L., \& Rowan-Robinson, M. 2010, MNRAS, 401, 35

Willmer, C. N. A. 1997 , AJ, 114, 898

Wu, Y., Shi, Y., Helou, G., et al. 2011, ApJ, 734, 40

Wyder, T. K., Treyer, M. A., Milliard, B., et al. 2005, ApJ, 619, L15

Xu, C. 2000, ApJ, 541, 134

Yuan, T.-T., Kewley, L. J., \& Sanders, D. B. 2010, ApJ, 709, 884

Zhu, G., Moustakas, J., \& Blanton, M. R. 2009, ApJ, 701, 86 